\title{
Istaroxime metabolite PST3093 selectively stimulates SERCA2a and reverses disease-induced changes in cardiac function
}

Martina Arici ${ }^{\mathrm{a} \#}$, Mara Ferrandi ${ }^{\mathrm{b} \#}$, Paolo Barassi ${ }^{\mathrm{b}}$, Shih-Che $\mathrm{Hsu}^{\mathrm{c}}$, Eleonora Torre ${ }^{\mathrm{a}}$, Andrea Luraghi $^{\mathrm{a}}$, Carlotta Ronchi ${ }^{\mathrm{a}}$, Gwo-Jyh Chang ${ }^{\mathrm{d}}$, Francesco Peri ${ }^{\mathrm{a}}$, Patrizia Ferrari ${ }^{\mathrm{b}}$, Giuseppe Bianchi $^{\mathrm{b}}$, Marcella Rocchetti ${ }^{\mathrm{a}}$ and Antonio Zaza ${ }^{\mathrm{a}^{*}}$

aDepartment of Biotechnology and Biosciences, Università degli Studi di Milano-Bicocca, Milan, Italy.

${ }^{b}$ Windtree Therapeutics Inc., Warrington, Pennsylvania, USA.

${ }^{\mathrm{c} C V i e}$ Therapeutics Limited, Taipei, Taiwan.

${ }^{\mathrm{d} C h a n g}$ Gung University, Tao-Yuan, Taiwan.

\# MA and MF contributed equally as first authors to the article

*MR and AZ contributed equally as senior authors to the article

\section{Corresponding authors:}

Antonio Zaza, MD, FESC.

Dipartimento di Biotecnologie e Bioscienze

Università degli Studi di Milano-Bicocca

P.za della Scienza 2, 2016 Milano (IT)

antonio.zaza@unimib.it

Marcella Rocchetti, PhD.

Dipartimento di Biotecnologie e Bioscienze

Università degli Studi di Milano-Bicocca

P.za della Scienza 2, 2016 Milano (IT)

marcella.rocchetti@unimib.it

\section{Category: Research Paper}




\section{Abstract}

Background. Heart failure (HF) therapeutic toolkit would strongly benefit from the availability of ino-lusitropic agents with a favorable pharmacodynamics and safety profile. Istaroxime is a promising agent, which combines $\mathrm{Na}^{+} / \mathrm{K}^{+}$pump inhibition with SERCA2a stimulation; however, it has a very short half-life and extensive metabolism to a molecule, named PST3093. The present work aims to investigate whether PST3093, still retains the pharmacodynamic properties of its parent compound. Methods and Results. We studied PST3093 for its effects on SERCA2a and $\mathrm{Na}^{+} / \mathrm{K}^{+}$ATPase activities, $\mathrm{Ca}^{2+}$ dynamics in isolated myocytes and hemodynamic effects in an in-vivo rat model of diabetic (streptozotocin (STZ)induced) cardiomyopathy. In cardiac microsomes, PST3093 did not inhibit the $\mathrm{Na}^{+} / \mathrm{K}^{+}$ATPase activity, but retained SERCA2a stimulatory activity. In in-vivo echocardiographic assessment, PST3093 improved overall cardiac performance (e.g. stroke volume) without decreasing heart rate, and reversed most STZ-induced abnormalities. Modulation of both systolic and diastolic indexes contributed to the improvement. For i.v. administration, PST3093 toxicity was considerably lower than that of istaroxime and its evaluation against 50 targets commonly involved in cardiac and extracardiac side-effects, failed to reveal significant interactions. Istaroxime infusion in patients led to accumulation of PST3093 in the plasma; clearance was substantially slower for PST3093 than for the parent compound. Conclusions. PST3093 is a "selective" SERCA2a activator, the prototype of a novel pharmacodynamic category with a potential in the ino-lusitropic approach to $\mathrm{HF}$, particularly with prevailing diastolic dysfunction. Its pharmacodynamics are peculiar and its pharmacokinetics are suitable to prolong the cardiac beneficial effect of istaroxime infusion. 


\section{Mechanism of action of PST3093}
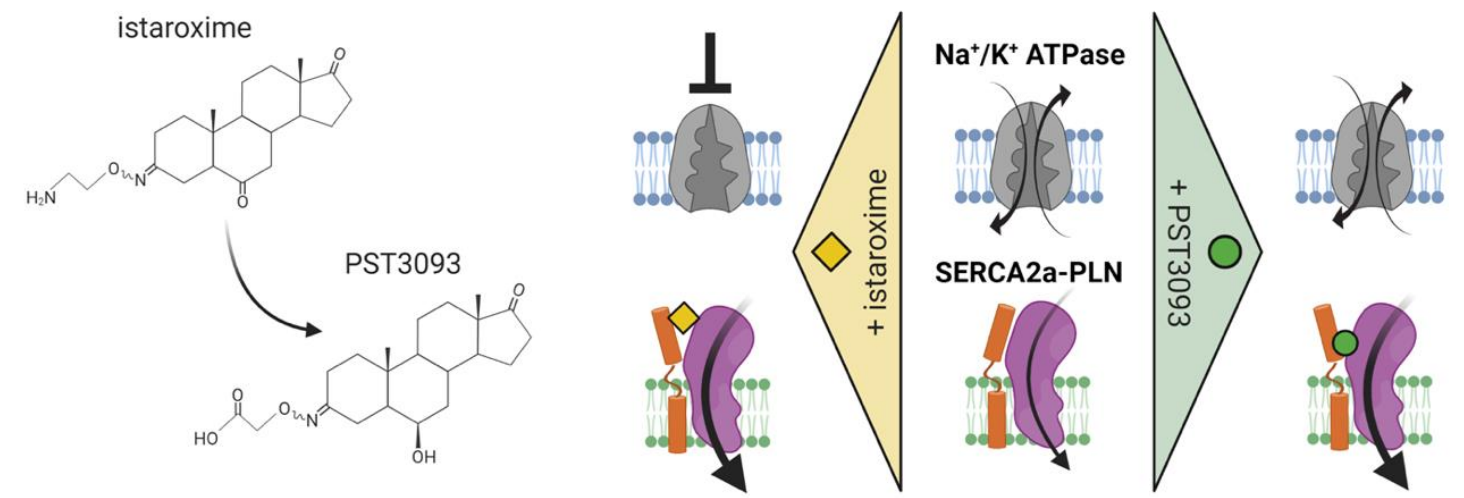

PST3093 in vivo effects in streptozotocin (STZ)-induced cardiomyopathy
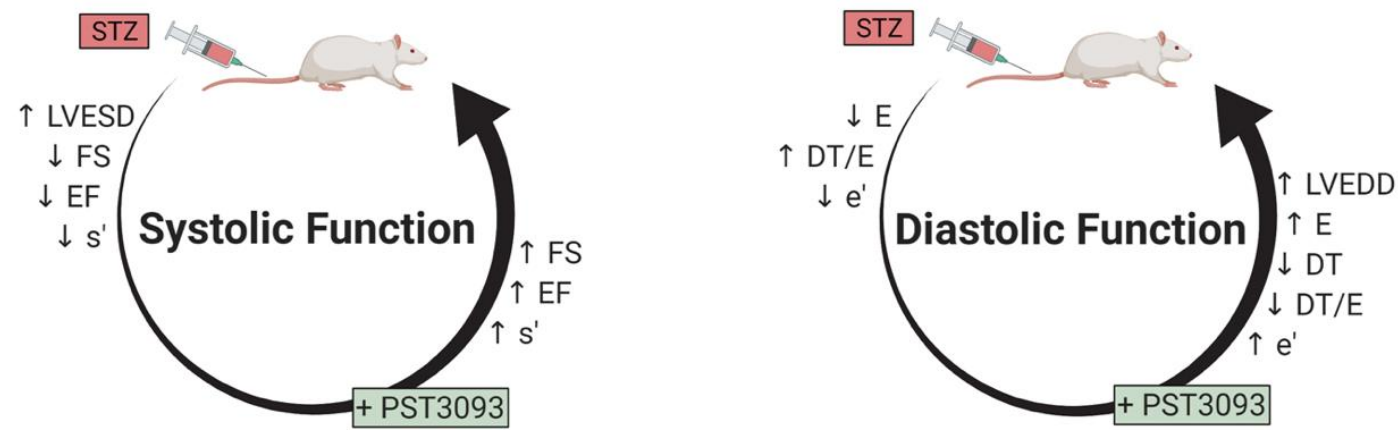

\section{Keywords}

istaroxime; metabolite; PST3093; heart failure; SERCA; inotropy-lusitropy.

Chemical compounds studied in this article: istaroxime (PubChem CID: 9841834); digoxin (PubChem CID: 2724385).

\section{Abbreviations}

AHF, acute heart failure; AP, action potential; APD, action potential duration; $\mathrm{AUC}_{\text {last }}$, the area under the drug plasma levels curve from the start of drug administration to the time of final sampling; Carest, resting $\mathrm{Ca}^{2+}$ at 20 s pause end; CasR, sarcoplasmic reticulum $\mathrm{Ca}^{2+}$ content; $\mathrm{CaT}, \mathrm{Ca}^{2+}$ transient; $\mathrm{C}_{\max }$, maximum observed concentration; $\mathrm{CO}$, cardiac output; $\mathrm{CPA}$, cyclopiazonic acid; DT, deceleration time; $\mathrm{E}_{\text {diast, }}$ diastolic potential; EF, ejection fraction; ER, 
excitation-release; $\mathrm{F}_{0}$, reference fluorescence; FS, fractional shortening; HF, heart failure; HR, heart rate; $\mathrm{I}_{\mathrm{CaL}} \mathrm{L}$ type $\mathrm{Ca}^{2+}$ current; $\mathrm{I}_{\mathrm{NaK}}, \mathrm{Na}^{+} / \mathrm{K}^{+}$ATPase current; i.p., intraperitoneal administration; i.v., intravenous administration; IVSTd or IVSTs, interventricular septum thickness in diastole or systole; $\mathrm{K}_{\mathrm{d}} \mathrm{Ca}, \mathrm{Ca}^{2+}$ dissociation constant, $\mathrm{LD}_{50}$, in vivo dose causing $50 \%$ mortality; LV, left ventricle; LVEDD or LVESD, LV end-diastolic or systolic diameter; $\mathrm{NCX}, \mathrm{Na}^{+} / \mathrm{Ca}^{2+}$ exchanger; PKs, pharmacokinetics; PLN, phospholamban; PWTd or PWTs, posterior wall thickness in diastole or systole; SERCA, sarcoplasmic reticulum $\mathrm{Ca}^{2+}$ ATPase; SR, sarcoplasmic reticulum; STV, short term variability; STZ, streptozotocin; SV, stroke volume; $\mathrm{SW}$, septal diastolic wall thickness; $\mathrm{T}_{0.5}, \mathrm{Ca}^{2+}$ transient decay half-time or elimination half life time; $\tau_{\text {decay }}$, time-constant of $\mathrm{Ca}^{2+}$ decay; TDI, Tissue Doppler Imaging; $\mathrm{T}_{\max }$, the time of maximum observed concentration; $\mathrm{V}_{\max }$, maximal hydrolytic velocity. 


\section{Introduction}

Heart failure (HF) is characterized by abnormal $\mathrm{Ca}^{2+}$ distribution among subcellular compartments, which contributes to impaired contractility and relaxation [1], facilitates arrhythmias [2] and, in the long run, contributes to myocardial remodeling [3]. Evidence of a deficient SERCA2a activity in HF dates to the 70's [4,5]. Since then, many studies confirmed this finding [6-8] showing that the impaired SERCA2a activity can often result from an overinhibition by phospholamban (PLN) [9,10]. Loss of SERCA2a function accounts for abnormal distribution of intracellular $\mathrm{Ca}^{2+}$ with numerous detrimental consequences. Interventions currently available to modulate myocyte $\mathrm{Ca}^{2+}$ handling (e.g. amines, PDE inhibitors etc.) stimulate SERCA2a, but they do so in the context of a multi-target action, thus resulting in untoward effects. Selective SERCA2a enhancement would afford inotropic and lusitropic effects without the drawbacks of the multi-target action [2]. Accordingly, the use of SERCA2a stimulation in HF therapy is receiving considerable attention and many attempts to selectively stimulate SERCA2a activity with gene therapy or "small molecule" agents have been reported [11-13]. Nonetheless, for reasons other than refutation of the principle, none of these attempts has been successfully translated into the clinic. The only exception is istaroxime, a smallmolecule drug, identified as SERCA2a enhancer by our group [14] and currently under clinical development for the treatment of acute HF $[15,16]$. Istaroxime has a double mechanism of action: it inhibits the $\mathrm{Na}^{+} / \mathrm{K}^{+}$pump [17] and activates SERCA2a [14]. Thus, istaroxime increases overall cell $\mathrm{Ca}^{2+}$ content while promoting rapid $\mathrm{Ca}^{2+}$ sequestration into the sarcoplasmic reticulum (SR). Notably, at variance with $\mathrm{Na}^{+} / \mathrm{K}^{+}$pump blockade alone, this neither facilitates spontaneous $\mathrm{Ca}^{2+}$ release from the SR [18], nor increases myocardial oxygen demand [19]. Thus, istaroxime may improve systolic and diastolic performance [15] without promoting arrhythmia or ischemia [16,20]. However, istaroxime has-a plasma half-life of less than 1 hour, because of extensive hepatic metabolism to a molecule, named PST3093 [16,20]; this restricts istaroxime usage to acute intravenous therapy. 
The present work aims to investigate whether PST3093 may, on its own, be endowed with pharmacological activity. To this end, PST3093 has been synthesized and compared to istaroxime and digoxin (as reference compounds) in experimental set-ups at different levels of biological organization and in the context of disease-induced dysfunction.

The data here reported indicate that PST3093 stimulates SERCA2a activity but, at variance with istaroxime (taken as reference compounds) it does not inhibit the $\mathrm{Na}^{+} / \mathrm{K}^{+}$ATPase. This pharmacodynamic profile translates to positive inotropy and lusitropy in an in-vivo disease model characterized by SERCA2a downregulation. Therefore, PST3093 qualifies as a "pure" SERCA2a activator, able to improve cardiac mechanical performance in-vivo.

\section{Methods}

Further detail on methods is given in the online supplement.

\subsection{Pharmacokinetics}

Pharmacokinetics (PKs) of istaroxime and its metabolite PST3093 was assessed in $30 \mathrm{HF}$ patients infused for 6 hours with istaroxime at $1.0 \mu \mathrm{g} / \mathrm{kg} / \mathrm{min}$ (secondary analysis of the HORIZON-HF study, NCT00616161) [20]. Blood samples were taken before, during, and up to 18 hours after starting the infusion. The lowest concentration resolved by the technique was $2.6 \mathrm{ng} \mathrm{mL}^{-1}$ for istaroxime and $2.9 \mathrm{ng} \mathrm{mL}^{-1}$ for PST3093, lower values were considered as zero. PKs parameters were estimated using the dedicated software Kinetica (version 4.4, Thermo Electron Corp., Waltham, Massachusetts). Samples were excluded from the analysis if contamination was suspected, or $\geq 2$ consecutive samples were missing. The following PKs parameters were estimated: maximum observed concentration $(\mathrm{Cmax})$, the time of maximum observed concentration (Tmax), the elimination half life time $\left(\mathrm{T}_{0.5}\right)$ and the area under the concentration curve from the start of istaroxime administration to the time of final sampling (AUClast). 


\subsection{Animal model}

All experiments involving animals conformed to the guidelines for Animal Care endorsed by the Milano-Bicocca and Chang Gung Universities and to the Directive 2010/63/EU of the European Parliament on the protection of animals used for scientific purposes.

Streptozotocin (STZ)-induced diabetes was selected as a pathological model because of its association with reduced SERCA2a function [21] and relevance to diastolic dysfunction [22], for which a lusitropic action may be more relevant. Diabetes was induced in Sprague Dawley male rats (150-175 g) by a single i.v. streptozotocin (STZ group, $50 \mathrm{mg} \mathrm{kg}{ }^{-1}$ ) injection. Control (healthy group) rats received STZ vehicle (citrate buffer). Fasting glycaemia was measured after 1 week and rats with values $>290 \mathrm{mg}^{-1} \mathrm{dL}^{-1}$ were considered diabetic [21]. Rats were euthanized by cervical dislocation under anesthesia with ketamine-xylazine (130-7.5 mg kg-1 i.p) 9 weeks after STZ injection.

\subsection{Biochemical measurements}

Total ATPase activity was assessed by measuring the rate of ${ }^{32} \mathrm{P}$-ATP release $\left(\mu \mathrm{mol} \cdot \mathrm{min}^{-1}\right)$ at $37^{\circ} \mathrm{C}$.

\subsection{1. $\mathrm{Na}^{+} / \mathrm{K}^{+}$ATPase activity assay}

The inhibitory effect of compounds was tested, at multiple concentrations, on suspensions of the enzyme $\alpha 1$ isoform from dog kidney by measuring ${ }^{32} \mathrm{P}$-ATP hydrolysis. $\mathrm{Na}^{+} / \mathrm{K}^{+}$ATPase activity was identified as the ouabain $(1 \mathrm{mM})$-sensitive component of total one; compound efficacy was expressed as the concentration exerting $50 \%$ inhibition ( $\left.\mathrm{IC}_{50}\right)$.

\subsubsection{SERCA ATPase activity assay}

Measurements were performed in whole tissue homogenates (rat) or in cardiac SR enriched microsomes (guinea-pig), including SERCA2a and PLN [21]. To test for PLN involvement in the effect of compounds, SERCA1 activity was also measured in PLN-free microsomes (from 
guinea-pig skeletal muscle) before and after reconstitution with the $\mathrm{PLN}_{1-32}$ inhibitory fragment at a ratio of 300:1 for PLN:SERCA. The SERCA component, identified as the cyclopiazonic acid (CPA, $10 \mu \mathrm{M})$-sensitive one, was measured at multiple $\mathrm{Ca}^{2+}$ concentrations (100-2000 $\mathrm{nM}$ ) as ${ }^{32} \mathrm{P}$-ATP hydrolysis [23] and $\mathrm{Ca}^{2+}$ dose-response curves were fitted to estimate SERCA maximal hydrolytic velocity $\left(\mathrm{V}_{\max } \mu \mathrm{mol} \cdot \mathrm{min}^{-1} \mathrm{mg}^{-1}\right.$ protein) and $\mathrm{Ca}^{2+}$ dissociation constant $\left(\mathrm{K}_{\mathrm{d}} \mathrm{Ca}, \mathrm{nM}\right)$. Either an increase of $\mathrm{V}_{\max }$, or a decrease of $\mathrm{K}_{\mathrm{d}} \mathrm{Ca}$ (increased $\mathrm{Ca}^{2+}$ affinity) stand for enhancement of SERCA function.

\subsubsection{PST3093 interaction with targets other than SERCA}

To predict potential off-target actions of PST3093, its interaction with a panel of 50 ligands, potentially relevant to off-target effects, was carried out by Eurofins (Taiwan) on crude membrane preparations according to Eurofins described procedures. PST3093 was tested at the concentration of $10 \mu \mathrm{M}$.

\subsection{Functional measurements in isolated myocytes}

To ensure stabilization of drug effect, effects on functional parameters were analyzed after incubating cells with PST3093 or vehicle (control) for at least $30 \mathrm{~min}$. Difference between means was thus tested by group comparison. All experiments were performed at $36.5^{\circ} \mathrm{C}$.

\subsection{1. $\mathrm{Na}^{+} / \mathrm{K}^{+}$ATPase current $\left(\mathrm{I}_{\mathrm{NaK}}\right)$}

$\mathrm{I}_{\mathrm{NaK}}$ was recorded in isolated rat left ventricular $(\mathrm{LV})$ myocytes as the holding current at -40 $\mathrm{mV}$ under conditions enhancing $\mathrm{I}_{\mathrm{NaK}}$ and minimizing contamination by other conductances $[21,24]$. $\mathrm{I}_{\mathrm{NaK}}$ inhibition by the compounds was expressed as percent reduction of ouabain (1 $\mathrm{mM}$ )-sensitive current; efficacy was expressed as the compound concentration exerting 50\% inhibition $\left(\mathrm{IC}_{50}\right)$ effect.

\subsubsection{Intracellular $\mathrm{Ca}^{2+}$ dynamics}

$\mathrm{Ca}^{2+}$-dependent fluorescence (Fluo 4-AM) was recorded in field stimulated (2 Hz) or patchclamped rat LV myocytes and quantified by normalized units $\left(\mathrm{F} / \mathrm{F}_{0}\right) . \mathrm{Ca}^{2+}$ dynamics was 
characterized through the properties of voltage-induced $\mathrm{Ca}^{2+}$ transients $(\mathrm{CaT}$, amplitude and decay kinetics) and caffeine-induced ones, the latter estimating total $\mathrm{SR} \mathrm{Ca}^{2+}$ content (CasR). Caffeine was applied 20 seconds after the last of a CaT sequence at $2 \mathrm{~Hz}$. Resting $\mathrm{Ca}^{2+}\left(\mathrm{Ca}_{\text {rest }}\right)$ was measured just before caffeine application to reflect the equilibrium value of cytosolic $\mathrm{Ca}^{2+}$. In patch-clamped myocytes, $\mathrm{SR} \mathrm{Ca}^{2+}$ uptake rate was evaluated through a "SR loading" voltage protocol, specifically devised to examine the system at multiple levels of $\mathrm{SR} \mathrm{Ca}^{2+}$ loading and to rule out $\mathrm{Na}^{+} / \mathrm{Ca}^{2+}$ exchanger (NCX) contribution. Current through L-type $\mathrm{Ca}^{2+}$ channel $\left(\mathrm{I}_{\mathrm{CaL}}\right)$ was simultaneously recorded and the excitation-release (ER) "gain" was calculated as the ratio between CaT amplitude and $\mathrm{Ca}^{2+}$ influx through $\mathrm{I}_{\mathrm{CaL}}$ up to $\mathrm{CaT}$ peak [14] (protocol in Figure S1).

\subsubsection{Electrical activity}

Action potentials (APs) were recorded (I-clamp) from isolated guinea-pig LV myocytes, selected because of the AP similarity to the human one [25], under Tyrode superfusion. AP duration at $50 \%$ and $90 \%$ repolarization $\left(\mathrm{APD}_{50}\right.$ and $\left.\mathrm{APD}_{90}\right)$ and diastolic potential $\left(\mathrm{E}_{\text {diast }}\right)$ were measured 1) during steady state pacing at several rates; 2) dynamically upon stepping between two rates (APD adaptation). During steady state pacing, short-term APD90 variability (STV) was calculated from 20-30 subsequent APD90 values according to Eq. 1 [26]:

$$
S T V=\sum(|A P D(n+1)-A P D n|) /[\text { nbeats } * \sqrt{ } 2]
$$

The kinetics of $\mathrm{APD}_{90}$ adaptation was quantified by the time constant $(\tau)$ of exponential data fitting.

\subsection{In-vivo studies}

\subsubsection{Acute drug toxicity in mice}

Acute toxicity of PST3093 was preliminarily evaluated in male Albino Swiss CD-1 mice by identifying the dose causing $50 \%$ mortality $\left(\mathrm{LD}_{50}, \mathrm{mg} \cdot \mathrm{kg}^{-1}\right.$ body weight $)$ at 24 hours after i.v. injection. PST3093 was dissolved in DMSO and injected at 50, 100, 200, and $250 \mathrm{mg} \mathrm{kg}^{-1}$ (4 
animals for each group). Higher PST3093 dose levels were not tested due to the solubility limit of the compound; control animals received the vehicle only (DMSO).

Acute toxicity of istaroxime in male CD1 mice was also evaluated for comparison, by testing i.v. dose levels of 15, 22, 27 and $33 \mathrm{mg} \mathrm{kg}^{-1}$ (5 animals for each group). Control animals received the vehicle only (saline).

\subsubsection{Hemodynamic studies in rats with diabetic cardiomyopathy}

Healthy and STZ rats were studied by transthoracic echocardiographic under urethane anesthesia (1.25 $\mathrm{gkg}^{-1}$ i.p.). Left-ventricular end-diastolic (LVEDD) and end-systolic (LVESD) diameter, posterior wall (PWT) and interventricular septal (IVST) thickness were measured according to the American Society of Echocardiography guidelines [27]. Fractional shortening was calculated as FS $=($ LVEDD-LVESD $) * \mathrm{LVEDD}^{-1}$. Trans-mitral flow velocity was measured (pulsed Doppler) to obtain early and late filling velocities (E, A waves) and $\mathrm{E}$ wave deceleration time (DT). DT was also normalized to E wave amplitude (DT/E ratio). Peak myocardial systolic (s') and diastolic velocities (e' and a'), were measured at the mitral annulus by Tissue Doppler Imaging (TDI).

According to our previous study [21], PST3093 was intravenously infused at $0.22 \mathrm{mg} \mathrm{kg}^{-1}(0.16$

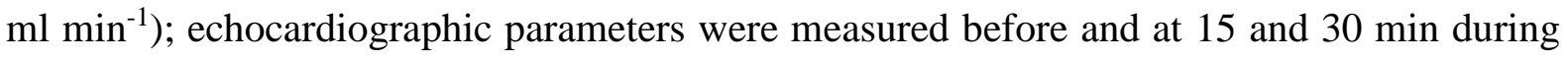
the infusion. Istaroxime $\left(0.22 \mathrm{mg} \cdot \mathrm{kg}^{-1}\right)$ and digoxin $\left(0.11 \mathrm{mg} \cdot \mathrm{kg}^{-1}\right)$, both infused for $15 \mathrm{~min}$, were used as comparators.

\subsection{Statistical analysis}

Individual means were compared by paired or unpaired $t$-test; multiple means were compared by one or two-way ANOVA for repeated measurements (RM) plus post-hoc Tukey's multiple comparisons; drug-induced changes in overall curve steepness were defined according to significance of the "factor X group" interaction. Data are reported as mean \pm SEM; $p<0.05$ 
defined statistical significance of differences in all comparisons. Number of animals $(\mathrm{N})$ and/or cells (n) are shown in each figure legend.

\subsection{Chemicals}

Istaroxime

\{PST2744:

[E,Z]-3-[(2-aminoethoxy)imino]-androstane-6,17-dione hydrochloride $\}$ and its metabolite PST3093 \{(E,Z)-[(6-beta-hydroxy-17-oxoandrostan-3ylidene)amino]oxyacetic acid\} were synthesized and produced at Prassis Research Institute and Sigma-Tau Pharmaceutical Company and then by CVie Therapeutics Limited and WindTree Therapeutics. The batch of PST3093 utilized for in-vitro and in-vivo studies was a 1:1 mixture of oxime E:Z isomers. It was synthesized according to standard procedures, characterized by NMR spectroscopy and its purity (about 95\%) was assessed by HPLC (Figure S2). Digoxin was purchased from Sigma-Aldrich.

\section{Results}

\subsection{Chemical structure of PST3093}

PST3093 is the final metabolite of istaroxime [20]; its chemical structure is shown in Figure 1A. Compared to istaroxime, PST3093 retains the oxime moiety at position 3 with the aminochain oxidized into a carboxylic chain, while the 6-keto group of istaroxime is stereo selectively reduced to a $6 \beta$-hydroxyl group.

\subsection{Pharmacokinetics (PKs)}

Figure 1B shows istaroxime and PST3093 plasma levels over a 6-hours istaroxime infusion at

$1 \mu \mathrm{g} \mathrm{kg}^{-1} \mathrm{~min}^{-1}$ and up to 12 hours after discontinuation of infusion. All patients showed measurable istaroxime levels until 10 min after stopping the infusion, drug levels decreased rapidly thereafter and just one patient had a quantifiable istaroxime level 2 hours after the end of the infusion. PST3093 plasma levels increased with a lag from the start of istaroxime 
infusion (as expected for a metabolite) being detectable in all patients from 1 hour after the start of infusion. Plasma PST3093 levels remained detectable long after discontinuation of the infusion, up to the last sample at 12 hours after wash out (Figure 1C). The data suggest that, if istaroxime infusion had continued beyond 6 hours, the metabolite would have accumulated further.

In quantitative terms, PST3093 had a plasma half-life $\left(\mathrm{T}_{0.5}\right)$ of about 9 hours, i.e. substantially longer than that of istaroxime (less than 1 hour), leading to a huge enhancement of the $\mathrm{AUC}_{\text {last }}$ index for PST3093; while the maximal observed concentration $\left(\mathrm{C}_{\max }\right)$ of PST3093 was similar to istaroxime one at this infusion rate, the time of its observation $\left(\mathrm{T}_{\max }\right)$ was longer for PST3093 in comparison to istaroxime (Figure 1C).

A

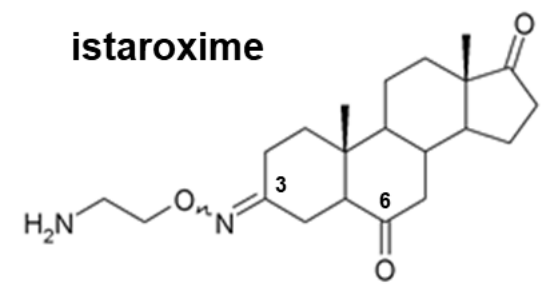

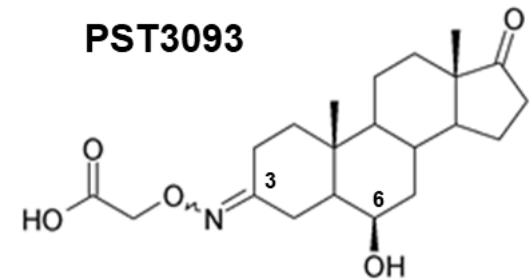

B

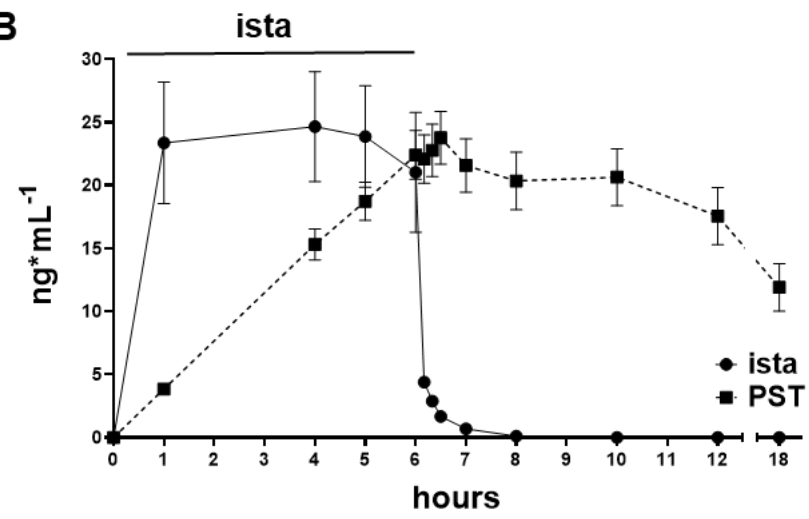

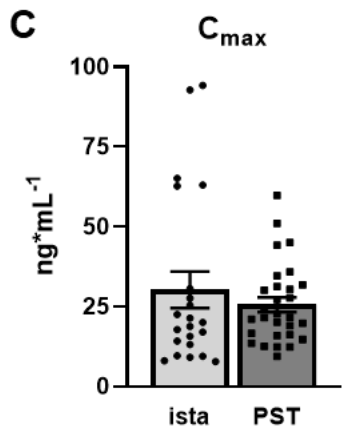

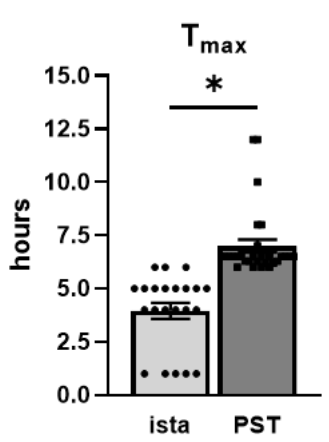

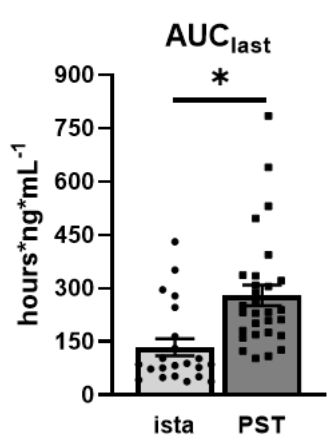

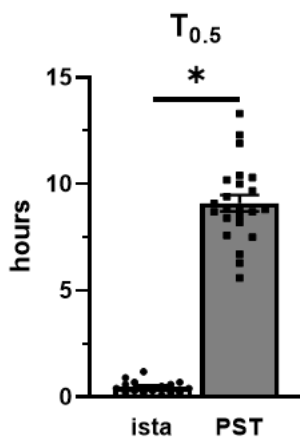


Figure 1. Chemical structure of istaroxime and its metabolite PST3093 (A) and PKs in humans (B-C). A) The main metabolic pathways of istaroxime are reduction of the carbonyl in position 6 catalyzed by carbonyl reductases and oxidative deamination of the primary amino group catalyzed by MAOs or tissue-bound semicarbazide-sensitive amine oxidase. B) Istaroxime ( $=22)$ and PST3093 ( $=29)$ plasma levels evaluated over 6 hours istaroxime infusion at $1 \mu \mathrm{g} / \mathrm{kg} / \mathrm{min}$ and up to 12 hours after wash-out. C) Statistics of the maximal observed concentration $\left(\mathrm{C}_{\max }\right)$, the time of $\mathrm{C}_{\max }\left(\mathrm{T}_{\max }\right)$, the area under the curve from the start of drug administration to the time of final sampling $\left(\mathrm{AUC}_{\text {last }}\right)$ and the plasma half-life $\left(\mathrm{T}_{0.5}\right)$; $* \mathrm{p}<0.05$ vs istaroxime (unpaired $t$-test). Data are the mean \pm SEM.

\subsection{Effect of PST3093 on $\mathrm{Na}^{+} / \mathrm{K}^{+}$ATPase}

\subsubsection{Effect on $\mathrm{Na}^{+} / \mathrm{K}^{+}$ATPase activity}

Compounds were tested in a range of concentrations from $10^{-9}$ to $10^{-4} \mathrm{M}$ (Figure $2 \mathrm{~A}$ ). $\mathrm{Na}^{+} / \mathrm{K}^{+}$ ATPase from dog kidney had a specific baseline activity of $14 \mu \mathrm{mol} \cdot \mathrm{min}^{-1} \mathrm{mg}^{-1}$ protein. The reference compound istaroxime inhibited $\mathrm{Na}^{+} / \mathrm{K}^{+}$ATPase activity with $\mathrm{IC}_{50}$ of $0.14 \pm 0.02 \mu \mathrm{M}$ in dog kidney (Figure 2A), which corresponds to a higher affinity as compared to that observed in rat renal preparations ( $\mathrm{IC}_{50}$ of $55 \pm 19 \mu \mathrm{M}$ from [21]). PST3093 did not inhibit the $\mathrm{Na}^{+} / \mathrm{K}^{+}$ ATPase activity up to $100 \mu \mathrm{M}$, the maximal tested concentration (Figure 2A).

\subsubsection{Effect on $\mathrm{Na}^{+} / \mathrm{K}^{+}$ATPase current $\left(\mathrm{I}_{\mathrm{NaK}}\right)$ in cardiac myocytes}

$\mathrm{I}_{\mathrm{NaK}}$ inhibition by PST3093 was evaluated in rat ventricular myocytes in comparison to istaroxime (Figure 2B). The estimated $\mathrm{IC}_{50}$ for $\mathrm{I}_{\mathrm{NaK}}$ inhibition by istaroxime was $32 \pm 4 \mu \mathrm{M}$, (from [21]); for PST3093 a barely detectable inhibition $(9.2 \pm 1.1 \%)$ was observed at the limit concentration for solubility $(100 \mu \mathrm{M})$, an effect that can be considered insignificant.

A

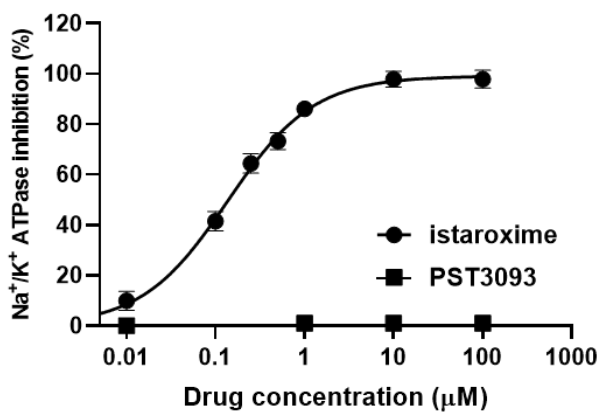

B $\quad \mathrm{Na}^{+} / \mathrm{K}^{+}$ATPase current $\left(\mathrm{I}_{\mathrm{NaK}}\right)$

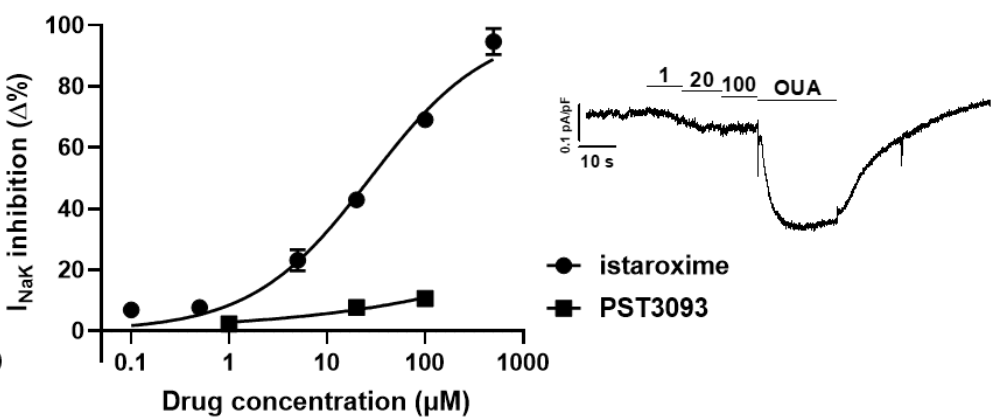

Figure 2. Modulation of $\mathrm{Na}^{+} / \mathrm{K}^{+}$ATPase activity. A) inhibition of $\mathrm{Na}^{+} / \mathrm{K}^{+}$ATPase activity by istaroxime and PST3093 in dog renal preparations $(\mathrm{N}=3)$; B) concentration-response curves 
for $\mathrm{I}_{\mathrm{NaK}}$ inhibition by PST3093 ( $\left.\mathrm{n}=15\right)$ and istaroxime (modified from [21]) in rat LV myocytes; $\mathrm{I}_{\mathrm{NaK}}$ recording under increasing concentrations of PST3093 and finally to ouabain (OUA as reference) is shown on the right. Data are the mean \pm SEM.

\subsection{Effect of PST3093 on SERCA ATPase activity}

\subsubsection{Effects on SERCA2a activity in normal and diseased myocardial preparations}

In STZ rat preparations ( $\mathrm{N}=30$ ), baseline SERCA2a $\mathrm{V}_{\max }$ was lower (by $-27 \%$ ) than in healthy ones $(\mathrm{N}=29)\left(0,199 \pm 0.01\right.$ vs $0,272 \pm 0.01 \mu \mathrm{mol}^{*} \mathrm{~min}^{-1} * \mathrm{mg}^{-1}$ protein, $\left.\mathrm{p}<0.05\right)$, with no difference in $\mathrm{K}_{\mathrm{d}} \mathrm{Ca}(448 \pm 35$ vs $393 \pm 22 \mathrm{nM}$, NS), similarly to what reported recently in the same setting [21]. As also reported previously [28], the response of enzyme kinetics parameters to modulation was species-specific: whereas in rat preparations both PST3093 and istaroxime (Figure 3) increased $\mathrm{V}_{\max }$, in guinea-pig ones the compounds decreased $\mathrm{K}_{\mathrm{d}} \mathrm{Ca}$ instead (Table S1).

Over the whole range of concentration tested $(0.1-1 \mu \mathrm{M})$, PST3093 and istaroxime failed to affect ATPase $\mathrm{Ca}^{2+}$-dependency in healthy rat preparations (Figure 3A,C), but similarly increased $\mathrm{V}_{\max }$ in $\mathrm{STZ}$ ones (e.g. $+22 \%$ and $+20 \%$, respectively at $300 \mathrm{nM}$ ) with thresholds at $100 \mathrm{nM}$ and $300 \mathrm{nM}$ for PST3093 and istaroxime, respectively (Figure 3B,D); SERCA2a $\mathrm{K}_{\mathrm{d}} \mathrm{Ca}$ in rat preparations was affected by neither istaroxime nor PST3093. Thus, PST3093 and istaroxime displayed similar potency in ameliorating disease-induced depression of SERCA2a ATPase activity.

PST3093 and istaroxime effect was also detected in preparations from normal guinea-pig hearts, where they both reduced the SERCA2a $\mathrm{K}_{\mathrm{d}} \mathrm{Ca}$ value by about $20 \%$ at $100 \mathrm{nM}$ (Table S1).

To summarize, PST3093 and istaroxime equally stimulated SERCA2a ATPase activity in preparations including PLN. Regardless of the kinetic parameter affected, a stimulatory effect was present in healthy guinea-pig microsomes and in rat homogenates from the STZ disease model. 


\section{SERCA2a ATPase activity}

Healthy

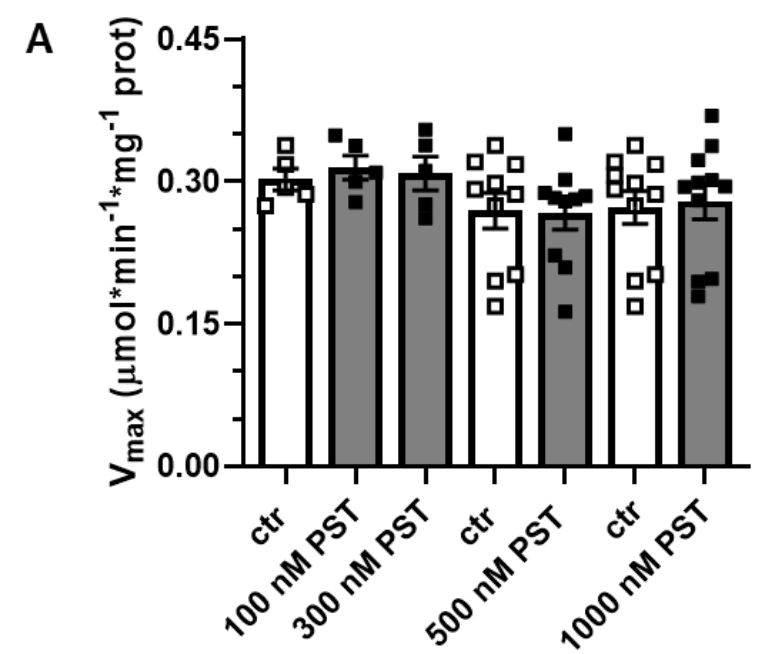

STZ
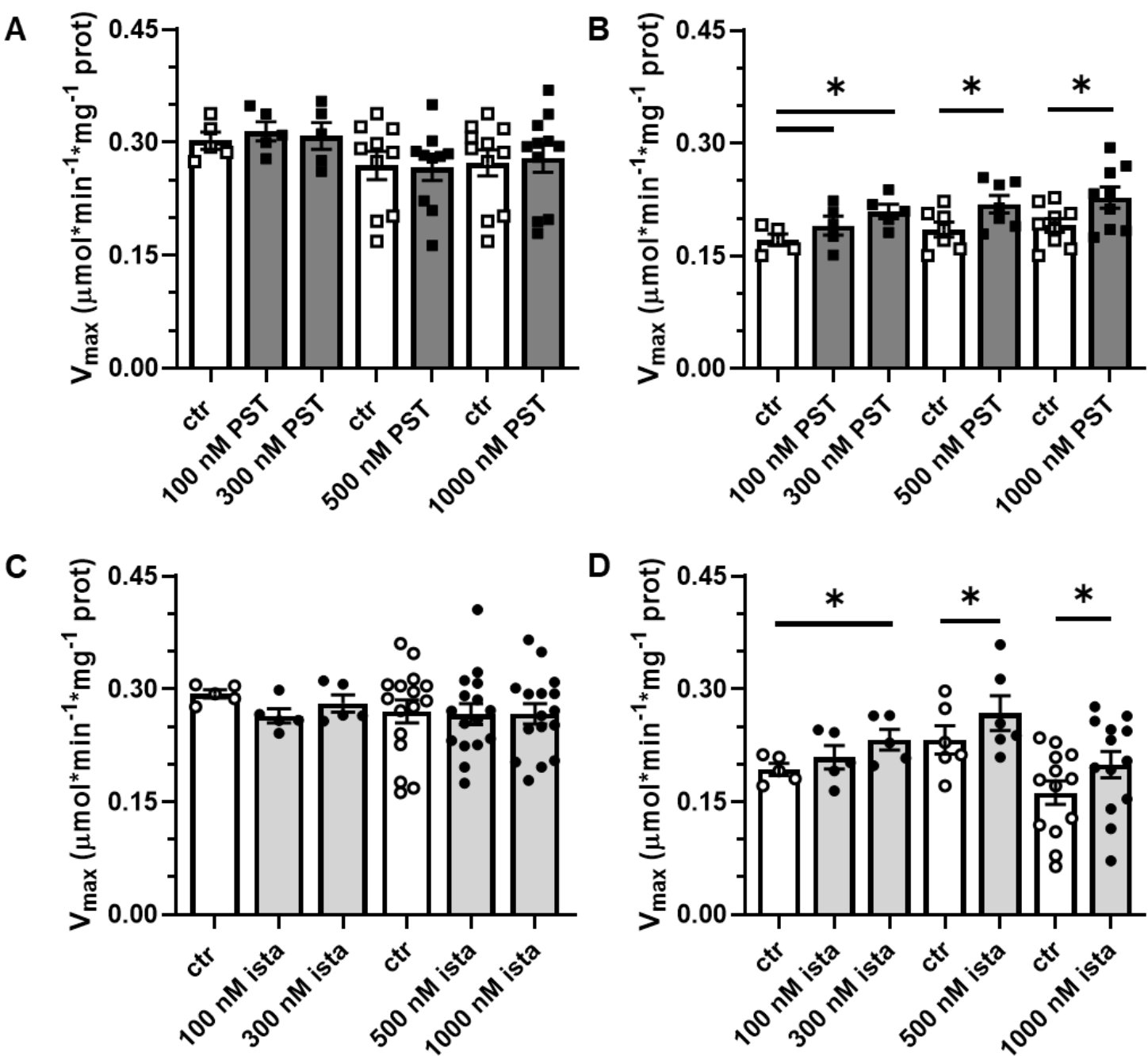

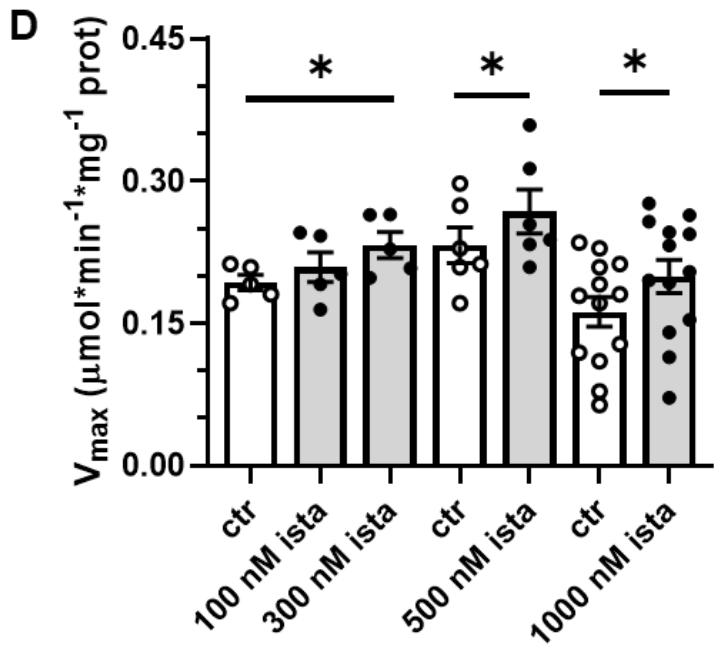

Figure 3. Modulation of SERCA2a ATPase activity in healthy and diseased (STZ) preparations. Effect of PST3093 ( $\mathrm{N}=5-11)$ (A-B) and istaroxime (N=5-16) (C-D) on SERCA2a $\mathrm{V}_{\max }$ estimated from $\mathrm{Ca}^{2+}$-dose response curves in cardiac homogenates from healthy and diabetic (STZ) rats. Internal controls (ctr) are provided. Data are the mean \pm SEM. * p $<0.05$ vs ctr (RM one-way ANOVA with post-hoc Tukey's multiple comparisons test or paired $t$-test).

\subsubsection{Dependency of SERCA stimulation by PST3093 on PLN}

In a range of concentrations from 30 to $1000 \mathrm{nM}$, PST3093 and istaroxime failed to affect SERCA1 activity in the absence of PLN in skeletal muscle preparations (Figure 4A,C). Reconstitution with the PLN $\mathrm{P}_{1-32}$ fragment markedly reduced SERCA1 affinity for $\mathrm{Ca}^{2+}\left(\mathrm{K}_{d} \mathrm{Ca}\right.$ increased by 23-26\%) (Figure 4B,D). Under this condition, both PST3093 (Figure 4B) and 
istaroxime (Figure 4D) dose-dependently reversed PLN-induced shift in $\mathrm{K}_{\mathrm{d}} \mathrm{Ca}$ with an $\mathrm{EC}_{50}$ of $39 \mathrm{nM}$ and $40 \mathrm{nM}$ respectively.

\section{SERCA1 ATPase activity}

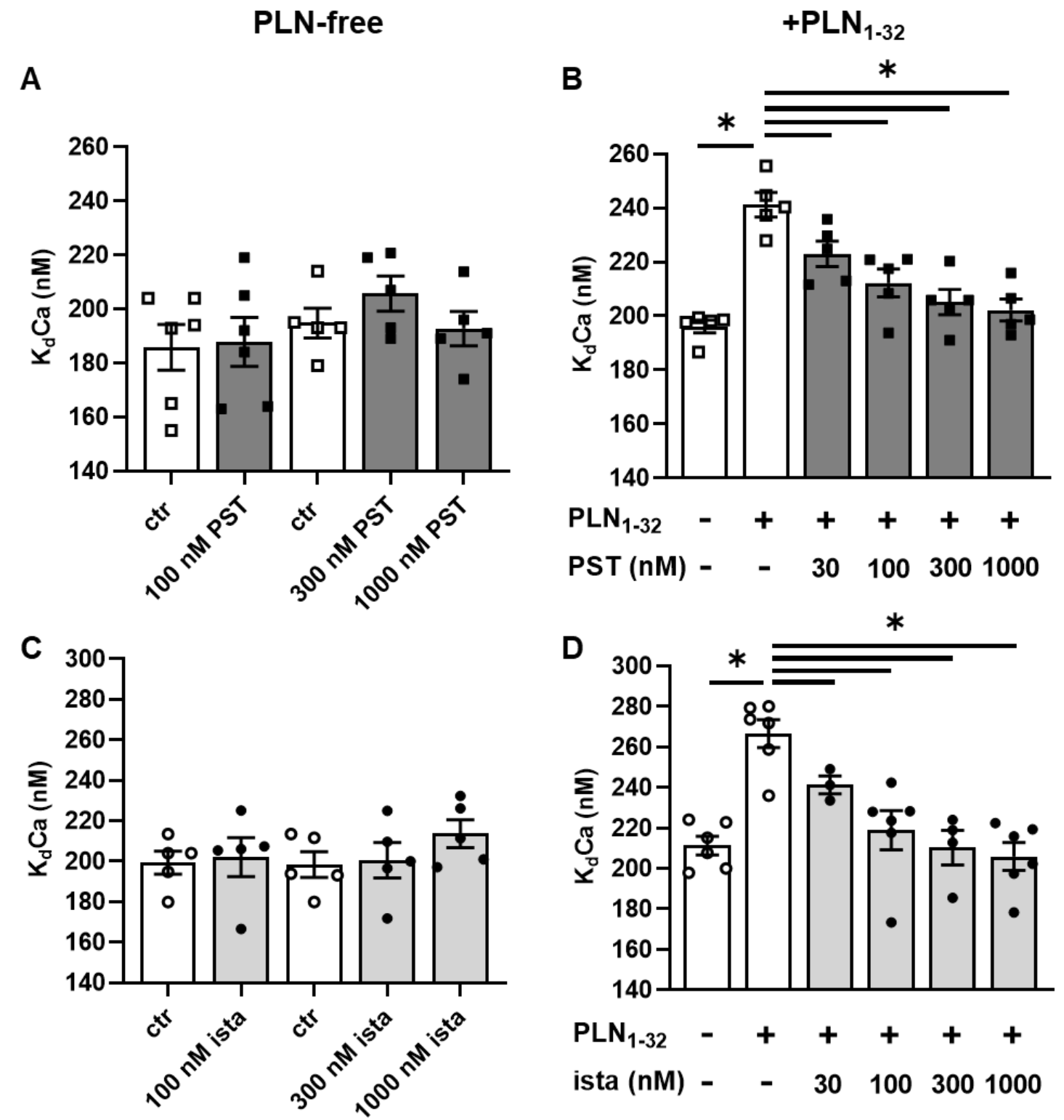

Figure 4. Modulation of SERCA1 ATPase activity. Concentration-dependency of PST3093 and istaroxime modulation of SERCA1 $\mathrm{K}_{d} \mathrm{Ca}$ in guinea-pig skeletal muscle microsomes containing SERCA1 alone $(\mathbf{A}, \mathbf{C})(\mathrm{N}=5,6)$ and after reconstitution with the $\mathrm{PLN}_{1-32}$ fragment $(B, D)(N=5,6)$. Data are the mean \pm SEM. ${ }^{*} \mathrm{p}<0.05$ (RM or mixed model one-way ANOVA plus post-hoc Tukey's multiple comparisons).

\subsection{PST3093 interaction with targets other than SERCA}

The targets panel (50 items) included membrane receptors, key enzymes, ion channels and transporters, relevant to potential off-target cardiac and extracardiac effects (list in Table S2); 
PST3093 was tested at the concentration of $10 \mu \mathrm{M}$. None among the 50 items met criteria for significance of interaction. Thus, at least for the ligands shown in Table S2, no off-target action of PST3093 is expected.

\subsection{Effects of PST3093 on intracellular $\mathrm{Ca}^{2+}$ dynamics in cardiac myocytes}

\subsubsection{Effect on intracellular $\mathrm{Ca}^{2+}$ dynamics under field-stimulation}

$\mathrm{Ca}^{2+}$ dynamics were analysed in field stimulated $(2 \mathrm{~Hz}$, Figure 5) rat LV myocytes isolated from healthy or STZ rats.

As already described in our previous work [21], STZ myocytes had a lower SR $\mathrm{Ca}^{2+}$ content (Cass) and a slower CaT decay than healthy ones; however, CaT amplitude remained unchanged. These changes are compatible with reduced SERCA2a function, possibly compensated by APD prolongation, known to increase cell $\mathrm{Ca}^{2+}$ content [21]. Whereas in healthy myocytes $1 \mu \mathrm{M}$ PST3093 failed to affect any of the $\mathrm{Ca}^{2+}$ dynamics parameters, in STZmyocytes PST3093 reduced the quiescence $\mathrm{Ca}^{2+}\left(\mathrm{Ca}_{\text {rest }}\right)$ prior to caffeine application and partially restored CasR and CaT decay. Moreover, PST3093 restored in STZ-myocytes the distribution of CasR values peculiar of healthy ones. Comparable results have been obtained with istaroxime at a concentration marginally affecting $\mathrm{Na}^{+} / \mathrm{K}^{+}$ATPase [21]. Taken together, this observation suggests that PST3093 improved $\mathrm{Ca}^{2+}$ sequestration into the SR during the post-train quiescence period. 


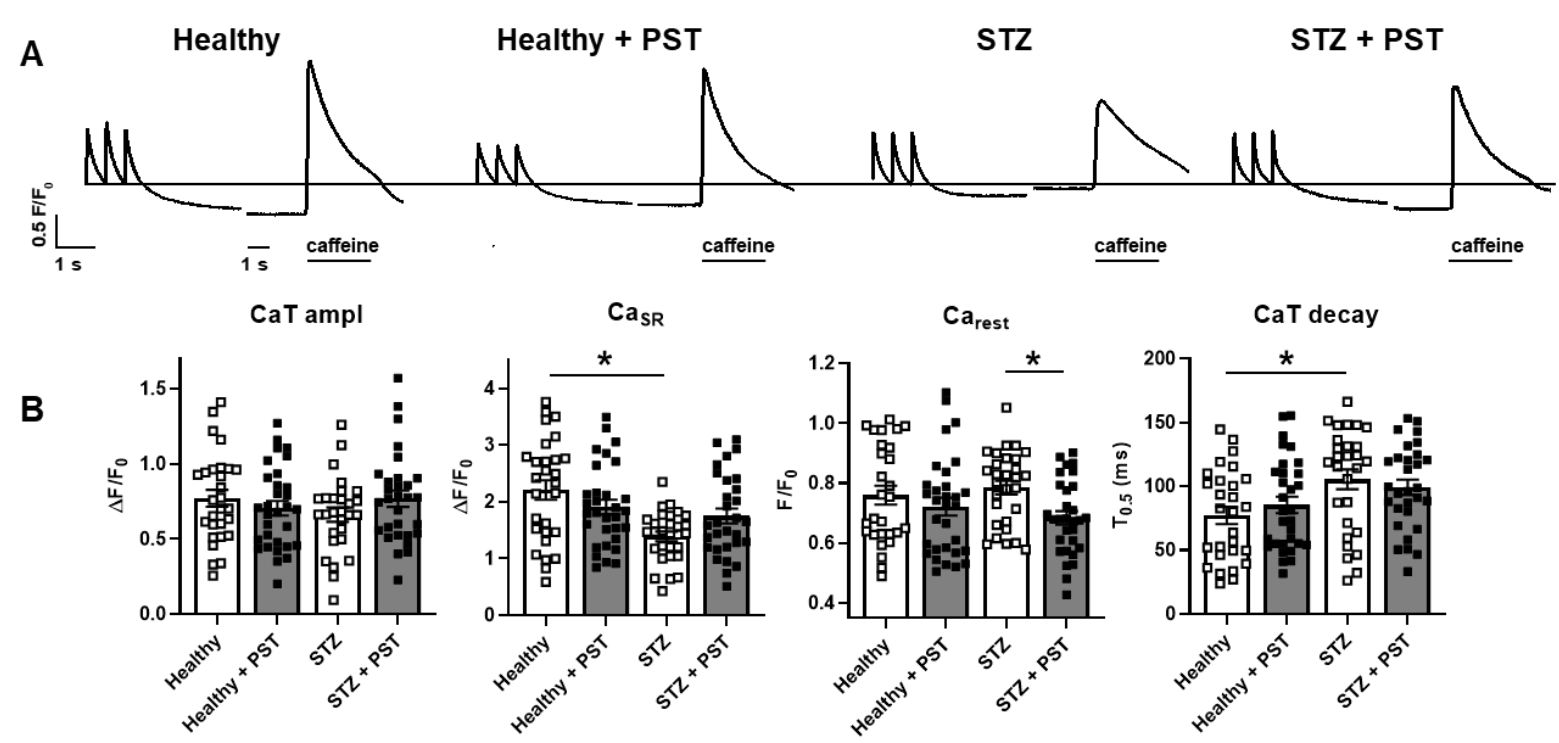

Figure 5. Modulation of intracellular $\mathrm{Ca}^{2+}$ handling in field stimulated myocytes from healthy and diseased (STZ) hearts. A) Representative recordings of $\mathrm{Ca}^{2+}$ transients (CaT) triggered by steady-state electrical stimulation at $2 \mathrm{~Hz}$ in intact cells, followed after 20 secs by caffeine-induced $\mathrm{Ca}^{2+}$ release measuring SR Ca ${ }^{2+}$ content $(\mathrm{CaSR})$. PST3093 $(1 \mu \mathrm{M})$ was tested in normal (healthy) and diseased (STZ) myocytes. B) Statistics for CaT amplitude, CasR, $\mathrm{Ca}^{2+}$ at pause end $\left(\mathrm{Ca}_{\text {rest }}\right)$, time for $50 \%$ CaT decay $\left(\mathrm{T}_{0.5}\right)$. CTR N=3 ( $\mathrm{n}=28$ w/o PST3093, $\mathrm{n}=31$ with PST3093), STZ N=4 ( $n=28$ w/o PST3093, $n=30$ with PST3093). * $\mathrm{p}<0.05$ (one-way ANOVA plus post-hoc Tukey's multiple comparisons).

\subsubsection{Effect on $S R \mathrm{Ca}^{2+}$ uptake function in V-clamped myocytes}

STZ-induced changes in repolarization affect $\mathrm{Ca}^{2+}$ handling in a direction masking SERCA2a downregulation [21]. Thus, the "SR loading" protocol (see methods and Figure S1) was performed under V-clamp and used to assess $\mathrm{SR} \mathrm{Ca}^{2+}$ uptake under conditions emphasizing SERCA2a role. In STZ myocytes, as compared to healthy ones, SR reloading was significantly depressed (both in terms of CaT amplitude and ER gain) and CaT decay was slower at all timepoints during reloading (Figure 6A). These changes are compatible with depressed SERCA2a function [21]. PST3093 $(1 \mu \mathrm{M})$ was tested in STZ myocytes (Figure 6B), where it sharply accelerated CaT decay, to restore the profile observed in healthy myocytes. Albeit less evident, drug-induced changes in CaT and ER gain pointed in the same direction. Comparable results have been obtained with istaroxime at a concentration marginally affecting $\mathrm{Na}^{+} / \mathrm{K}^{+}$ATPase [21]. 
Overall, PST3093 restored SR function in diseased myocytes, most likely through SERCA2a enhancement, within the context of an intact cellular environment.

A

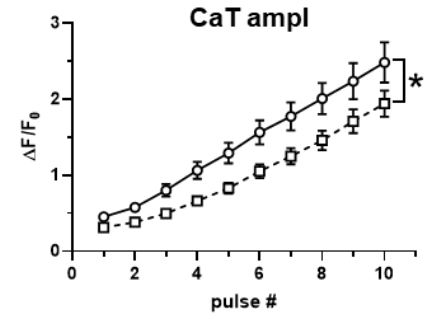

B

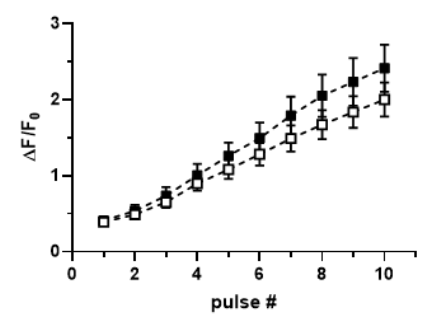

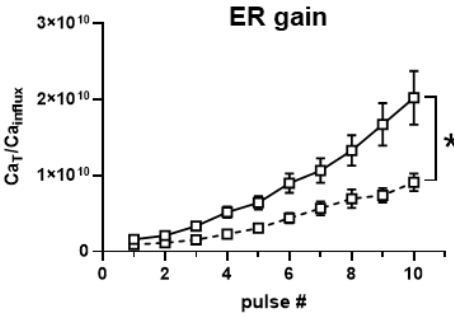
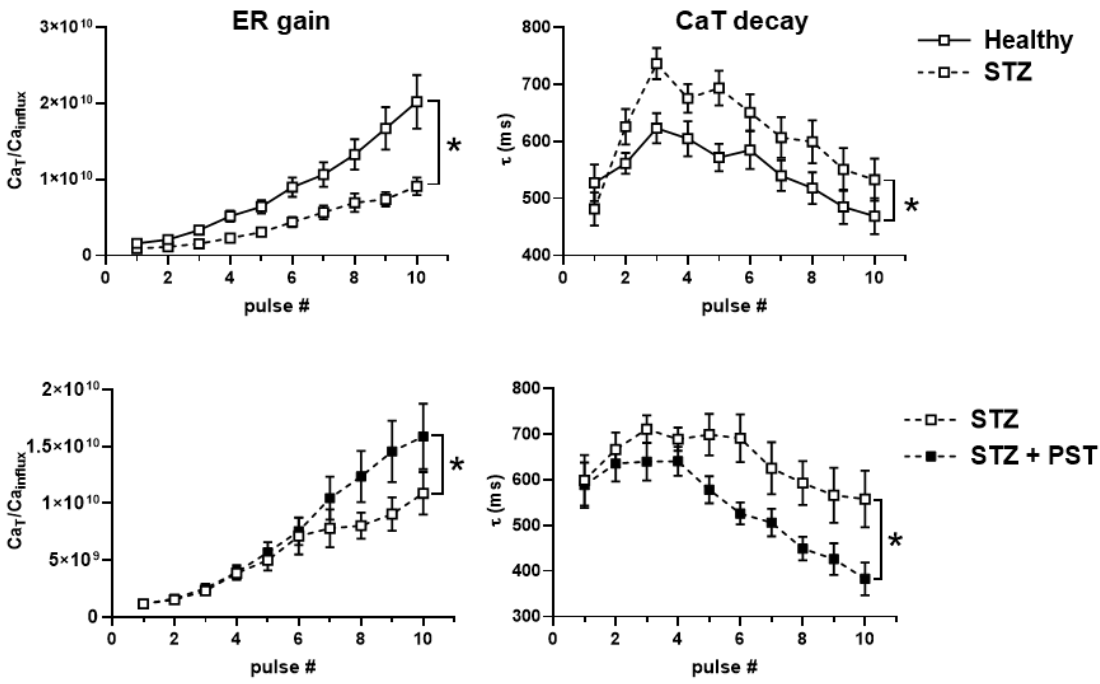

Figure 6. Modulation of SR $\mathrm{Ca}^{2+}$ uptake under $\mathrm{NCX}$ inhibition in V-clamped myocytes from STZ hearts. A) Disease (STZ) effect on SR $\mathrm{Ca}^{2+}$ loading in patch-clamped myocytes. SR Ca ${ }^{2+}$ loading by a train of V-clamp pulses was initiated after caffeine-induced SR depletion; NCX was blocked by $\mathrm{Na}^{+}$substitution to identify SERCA2a-specific effects (see Methods and Figure $\mathrm{S} 1)$; myocytes from healthy $(\mathrm{N}=9, \mathrm{n}=32)$ and diseased hearts (STZ, $N=6, n=31)$ are compared. B) PST3093 effect in STZ myocytes (N=4, w/o PST3093 n=18, with PST3093, $\mathrm{n}=19$ ). Panels from left to right: CaT amplitude, Excitation-Release (ER) gain (the ratio between $\mathrm{CaT}$ amplitude and $\mathrm{Ca}^{2+}$ influx through $\mathrm{I}_{\mathrm{CaL}}$ ), time constant $(\tau)$ of $\mathrm{CaT}$ decay. ${ }^{*} \mathrm{p}<0.05$ for the "interaction factor" in two-way ANOVA for repeated measurements, indicating a different steepness of curves.

\subsection{Effects of PST3093 on cellular electrical activity}

To assess the electrophysiological safety of PST3093, its effects on AP of LV myocytes were investigated. Guinea-pig myocytes were used, instead of rat ones, because their AP is closer to the human one.

PST3093 (100 nM) marginally reduced APD50 at all pacing rates, leaving the other AP parameters unchanged (Figure 7A-B). Notably, also APD rate-dependency at steady-state and the kinetics of APD adaptation following a step change in rate, were unaffected by the agent (Figure 7C). STV of APD90, a reporter of repolarization stability, was also unaffected by 
PST3093 at all pacing rates (Figure 7D-E). Except for the absence of APD $_{50}$ reduction, similar results were obtained with PST3093 at $1 \mu \mathrm{M}$ (Figure S3).

The paucity of PST3093 effects on the AP is consistent with the absence of hits in the analysis of PST3093 interaction (up to $10 \mu \mathrm{M}$ ) with molecular targets other than SERCA2a, among them ion channels and transporters (Table S2).

A

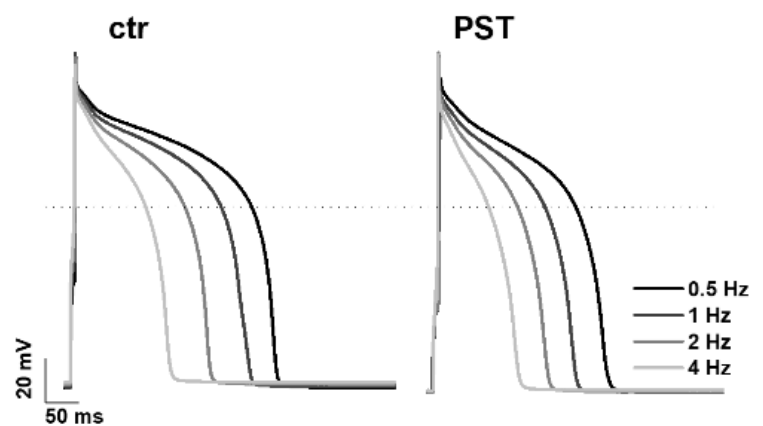

B

$E_{\text {diast }}$

$\mathrm{APD}_{50}$

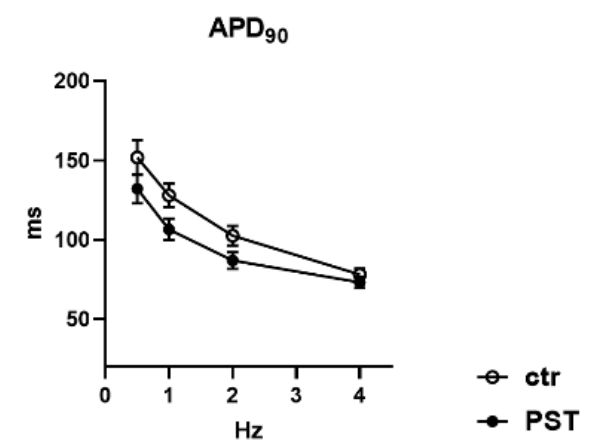

\section{C $\quad \mathrm{APD}_{90}$ adaptation kinetic}
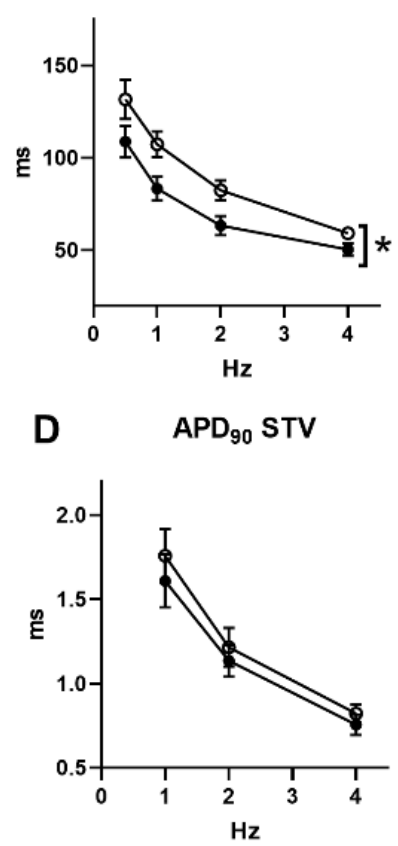

E
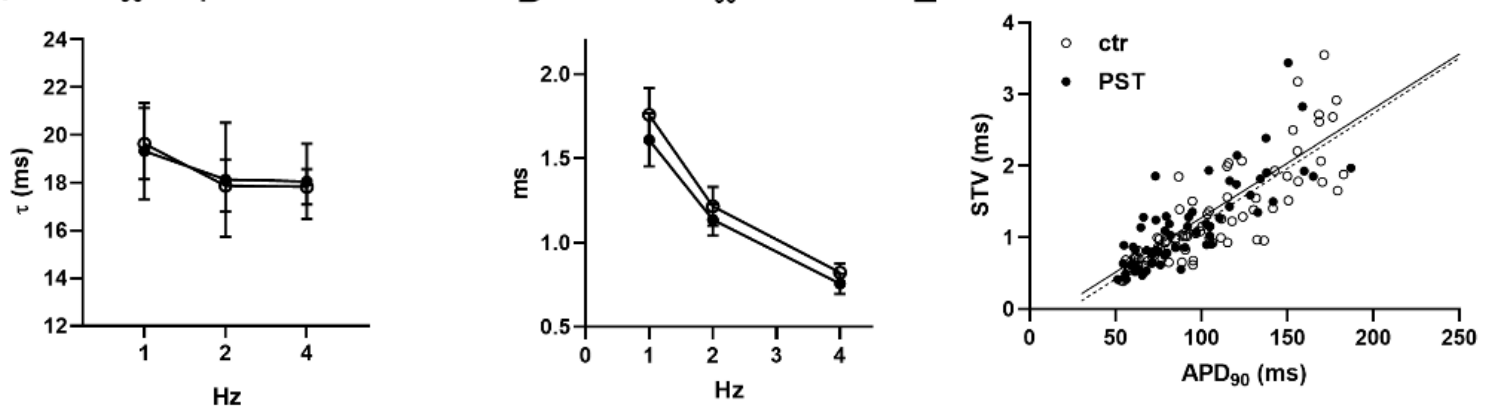

Figure 7. Modulation of electrical activity in guinea pig myocytes. The effect of PST3093 $100 \mathrm{nM}$ was tested on action potential (AP) parameters and their steady-state rate-dependency in guinea pig myocytes $(\mathrm{N}=5)$. A) representative APs recorded at $0.5,1,2,4 \mathrm{~Hz}$ in control (left) and with $100 \mathrm{nM}$ PST3093 (right). B) Effect on the rate dependency of diastolic potential ( $\left.E_{\text {diast }}\right)$ and AP duration (APD s0 $\left._{1}, A_{90}\right)(n \geq 24$ w/o PST3093, $n \geq 22$ with PST3093). C) Effect on the time constant $(\tau)$ of $\mathrm{APD}_{90}$ adaptation following a step change in rate $(\mathrm{n} \geq 22 \mathrm{w} / \mathrm{o}$ PST3093, $\mathrm{n} \geq 16$ with PST3093). D) Effect on the rate-dependency of APD 90 short term variability (STV) ( $\mathrm{n} \geq 24$ w/o PST3093, $\mathrm{n} \geq 20$ with PST3093). E) Effect on the correlation between STV of $\mathrm{APD}_{90}$ and mean $\mathrm{APD}_{90}$; data from 1, 2, $4 \mathrm{~Hz}$ were pooled. ${ }^{*} \mathrm{p}<0.05$ for the "interaction factor" of RM two-way ANOVA. The effect of PST3093 at a higher concentration $(1 \mu \mathrm{M})$ is reported in Figure $\mathrm{S} 3$. 


\subsection{In vivo acute toxicity in mice}

In vivo toxicity after i.v. injection was investigated in mice for PST3093 and istaroxime. PST3093 was well tolerated and did not cause death up to $250 \mathrm{mg} / \mathrm{kg}$. The $\mathrm{LD}_{50}$ was not calculated since no deaths occurred at the maximal usable dose (limited by solubility). The $\mathrm{LD}_{50}$ for istaroxime was $23.06 \mathrm{mg} / \mathrm{kg}$. The main signs of toxicity were prostration, gasping and convulsions. In most of the animals, death occurred within 5 min after istaroxime administration. Post-mortem examination revealed pulmonary oedema and/or haemorrhages and generalized organ congestion. No remarkable alterations were found in the surviving animals.

Therefore, when intravenously administered, PST3093 was far less toxic than istaroxime, a result ascribable to its lack of effects on the $\mathrm{Na}^{+} / \mathrm{K}^{+}$ATPase.

\subsection{Modulation of cardiac function in vivo in rats with diabetic cardiomyopathy}

\subsubsection{Features of the disease model}

Fasting hyperglycaemia, polydipsia, polyuria and polyphagia ensued 1 week after STZ injection; none of these symptoms was observed in healthy rats. Eight weeks after STZ, total body weight (BW) was substantially lower in STZ rats; LV mass (by echo) was reduced in absolute value but, when normalized to BW, was not significantly different in STZ rats (Table S3). The STZ model was completely characterized in a previous work of ours [21].

In this study a comprehensive echocardiographic analysis of STZ rats in comparison to healthy ones was performed. Figure 8 compares some echo indexes in STZ vs healthy rats; Table S4 lists all the measured echo parameters in the two groups. Heart rate (HR) was lower in STZ rats $(-20 \% ; \mathrm{p}<0.05)$ and stroke volume $(\mathrm{SV})$ unchanged; nonetheless, differences in cardiac output (CO) between the two groups did not achieve significance. Systolic indexes: in STZ rats LV end-systolic diameter (LVESD) was larger and the ejection fraction (EF) reduced; 
fractional shortening (FS) and systolic tissue velocity (s') were depressed. Diastolic indexes: in STZ rats LV end-diastolic diameter (LVEDD) tended to be larger; peak E wave velocity (E) was slightly smaller and A wave velocity (A) was unchanged (E/A unchanged); E wave deceleration time (DT) was unchanged in absolute, but the DT/E ratio increased. Changes in early (e') and late (a') diastolic tissue velocities paralleled those in E and A waves; therefore, the e'/a' and E/e' ratios did not differ between STZ and healthy rats.

To summarize, in STZ rats echocardiographic abnormalities were rather subtle; nonetheless, 11 out of 21 indexes were significantly affected indicating derangements in both systolic and diastolic function.
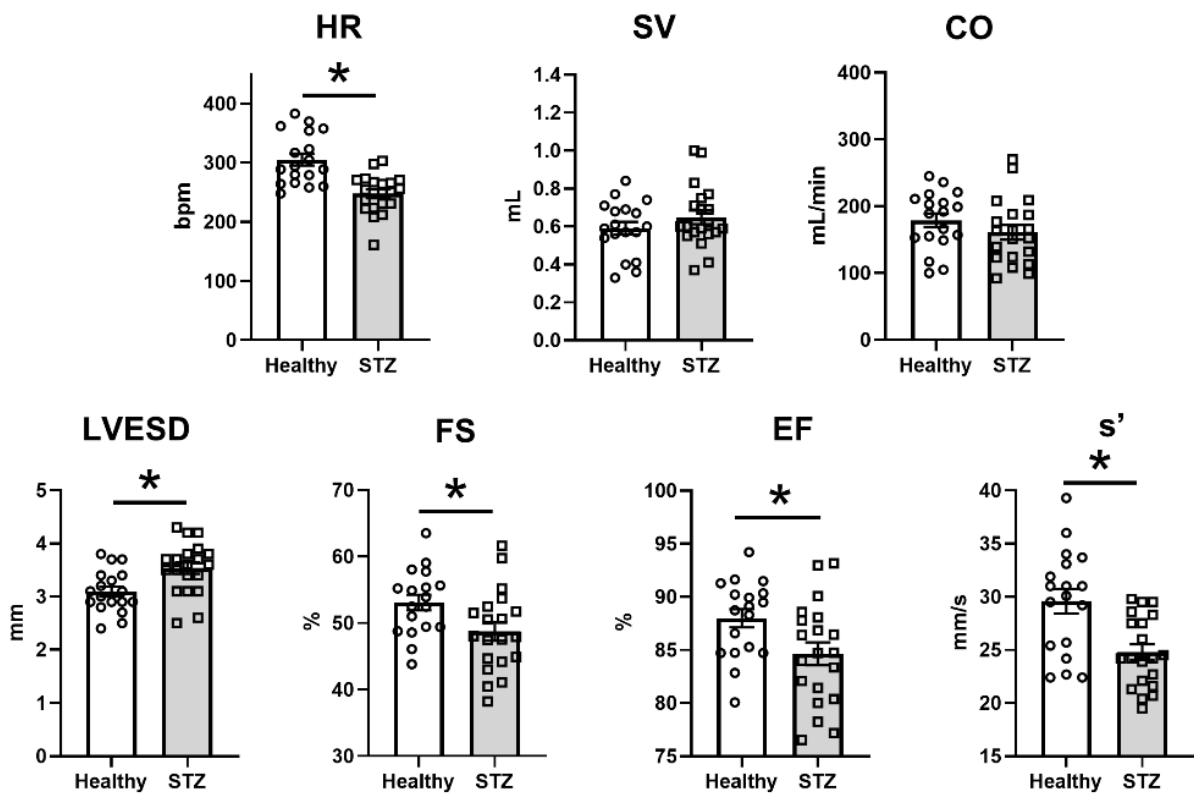

LVEDD

E

DT
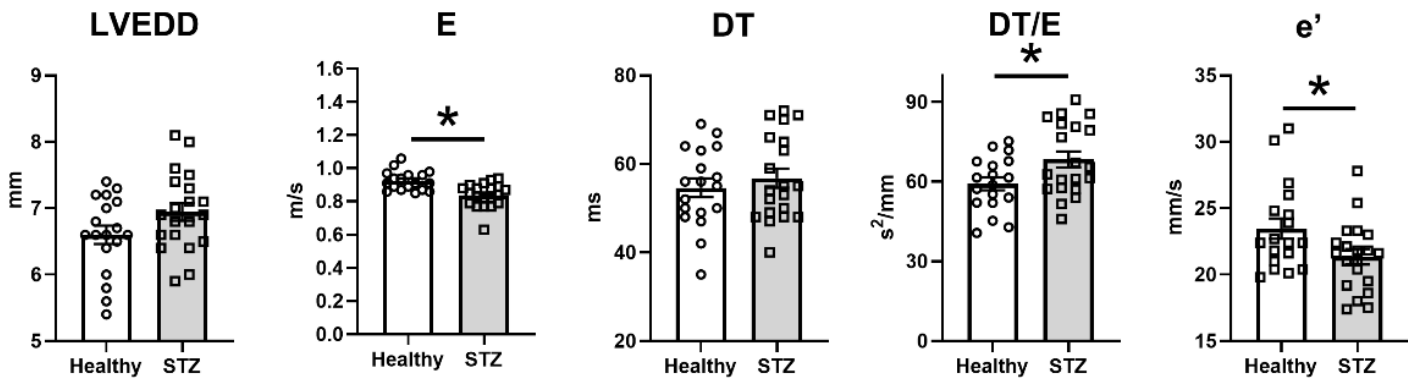

Figure 8. Disease (STZ) effect on in-vivo echocardiographic parameters. Echocardiographic parameters are compared between healthy rats $(\mathrm{N}=18)$ and 8 weeks after STZ treatment $(\mathrm{N}=20)$. Top row, global function parameters: HR: heart rate; SV: stroke volume; CO: cardiac output. Mid row, systolic function parameters: LVESD: left ventricular end-systolic diameter; FS: fractional shortening; EF: ejection fraction; s': peak systolic tissue velocity. Bottom row, diastolic function parameters: LVEDD: left ventricular end-diastolic 
diameter; E: E wave amplitude; DT: E wave deceleration time; DT/E: normalized DT; e': peak early diastolic tissue velocity. ${ }^{*} \mathrm{p}<0.05$ (unpaired $t$-test).

\subsubsection{Drug effects in the disease model}

The in vivo acute effect of PST3093 $\left(0.22 \mathrm{mg} \cdot \mathrm{kg}^{-1} \mathrm{~min}^{-1}\right)$ on echo indexes of STZ rats was investigated at 15 and 30 minutes of infusion (Figure 9). Data at 15 min were also obtained with istaroxime $\left(0.22 \mathrm{mg} \cdot \mathrm{kg}^{-1} \cdot \mathrm{min}^{-1}\right)$ and digoxin $\left(0.11 \mathrm{mg} \cdot \mathrm{kg}^{-1} \cdot \mathrm{min}^{-1}\right)$ (Table 1).

Overall, PST3093 increased SV volume and, albeit unchanged HR, it increased CO (Figure 9). Systolic indexes: PST3093 tended to decrease LVESD and increased FS, EF and s'. Diastolic indexes: PST3093 increased LVEDD, E, A and e' (E/A and E/e' unchanged, Table 1); DT and DT/E were reduced. PST3093 effect was almost complete at 15 min of infusion, only minor increments were observed at $30 \mathrm{~min}$ (Figure 9). Collectively, PST3093 improved overall cardiac function, both systolic and diastolic, beyond simple recovery of STZ-induced derangements. As shown in Figure 9, PST3093 "reversed" STZ-induced changes in 7 out of 11 indexes; moreover, five additional indexes, unaffected by STZ, were changed by PST3093 in a direction compatible with positive inotropy/lusitropy (Table 1).

PST3093 effects were only partially shared by istaroxime at the same infusion rate (Table 1). Istaroxime failed to increase SV and systolic indexes (SV, FS, EF, s'); it increased CO but, at variance with PST3093, this was because HR increased. Similar to PST3093, istaroxime shortened DT, DT/E and increased e', a' and A; however, it did not change E, thus reducing E/e'. Qualitatively similar results were obtained in STZ rats acutely treated with 0.11 $\mathrm{mg} / \mathrm{kg} / \mathrm{min}$ istaroxime [21].

Digoxin (Table 1), as expected from its inotropic effect, increased systolic indexes (FS, EF and s'); however, at variance with PST3093, it did not affect SV or CO. Notably, also digoxin improved diastolic function to some extent: E/A, DT and DT/E were reduced and e' was increased. 


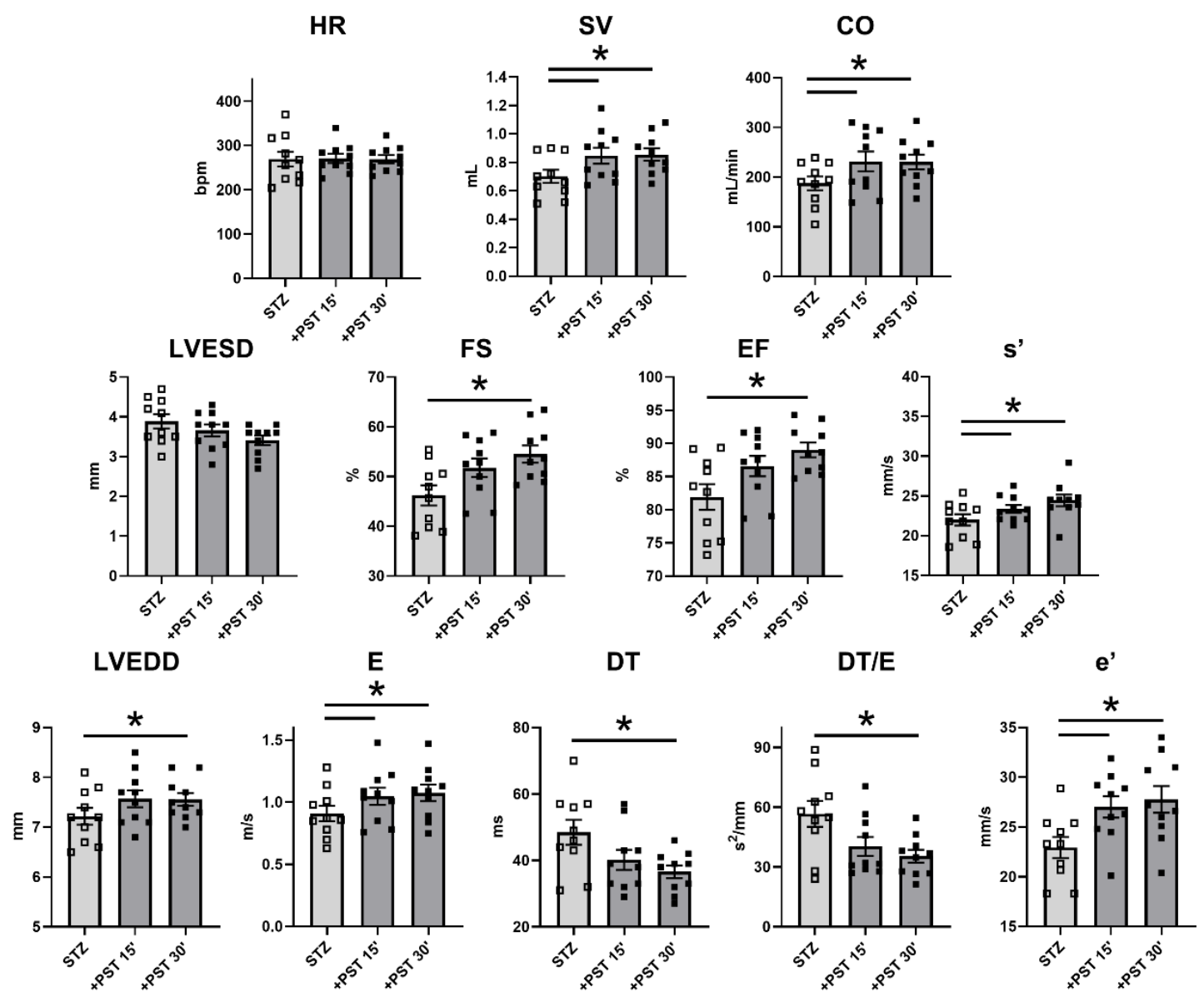

Figure 9. PST3093 effects on in-vivo echocardiographic parameters in diseased (STZ) rats. PST3093 was intravenously infused $\left(0.22 \mathrm{mg} \cdot \mathrm{kg}^{-1} \cdot \mathrm{min}^{-1}\right)$ in rats 8 weeks after STZ treatment. Echocardiographic parameters were measured before and at 15 and 30 min during drug infusion. Rows and symbols as in Figure 8; $\mathrm{N}=10$ rats; ${ }^{*} \mathrm{p}<0.05$. Data are the mean \pm SEM. *p<0.05 (RM one-way ANOVA plus post-hoc Tukey's multiple comparisons). Effects of PST3093 on all echocardiographic parameters at 15 minutes of infusion in STZ rats in comparison to istaroxime and digoxin are reported in Table 1. 
Table 1: Effects of PST3093, istaroxime and digoxin on echo indexes of STZ rats at 15 minutes of infusion. Data are the mean \pm SEM. $\# \mathrm{p}<0.05$ vs before drug infusion (paired $t$ test).

\begin{tabular}{|c|c|c|c|c|c|c|c|}
\hline \multicolumn{2}{|c|}{ Echo parameters } & \multicolumn{2}{|c|}{$\begin{array}{c}\text { Istaroxime } \\
0,22 \mathrm{mg} / \mathrm{kg} / \mathrm{min}\end{array}$} & \multicolumn{2}{|c|}{$\begin{array}{c}\text { PST3093 } \\
0,22 \mathrm{mg} / \mathrm{kg} / \mathrm{min}\end{array}$} & \multicolumn{2}{|c|}{$\begin{array}{c}\text { Digoxin } \\
0,11 \mathrm{mg} / \mathrm{kg} / \mathrm{min}\end{array}$} \\
\hline & & before & after $15 \mathrm{~min}$ & before & after $15 \mathrm{~min}$ & before & after $15 \mathrm{~min}$ \\
\hline \multirow{6}{*}{$\begin{array}{l}\text { Morphometric } \\
\text { parameters }\end{array}$} & IVSTd (mm) & $1,91 \pm 0,09$ & $1,97 \pm 0,16$ & $1,89 \pm 0,09$ & $1,86 \pm 0,11$ & $1,79 \pm 0,07$ & $1,81 \pm 0,04$ \\
\hline & PWTd (mm) & $1,6 \pm 0,07$ & $1,68 \pm 0,09$ & $1,46 \pm 0,05$ & $1,47 \pm 0,07$ & $1,71 \pm 0,08$ & $1,66 \pm 0,1$ \\
\hline & LVEDD (mm) & $7,25 \pm 0,15$ & $7,1 \pm 0,2$ & $7,22 \pm 0,17$ & $7,57 \pm 0,17$ & $6,76 \pm 0,15$ & $6,67 \pm 0,15$ \\
\hline & IVSTs (mm) & $2,4 \pm 0,1$ & $2,45 \pm 0,12$ & $2,16 \pm 0,11$ & $2,31 \pm 0,13$ & $2,25 \pm 0,14$ & $2,31 \pm 0,1$ \\
\hline & PWTs (mm) & $2,33 \pm 0,16$ & $2,42 \pm 0,15$ & $2,43 \pm 0,12$ & $2,62 \pm 0,14$ & $2,47 \pm 0,16$ & $2,68 \pm 0,14$ \\
\hline & LVESD (mm) & $3,82 \pm 0,21$ & $3,8 \pm 0,2$ & $3,89 \pm 0,18$ & $3,66 \pm 0,15$ & $3,53 \pm 0,17$ & $3,14 \pm 0,14 \#$ \\
\hline \multirow{3}{*}{$\begin{array}{c}\text { Systolic } \\
\text { parameters }\end{array}$} & FS (\%) & $47,21 \pm 2,4$ & $46,72 \pm 2,1$ & $46,2 \pm 2,02$ & $51,8 \pm 1,89 \#$ & $47,62 \pm 1,9$ & $53,09 \pm 1,8 \#$ \\
\hline & $\mathrm{s}^{\prime}(\mathrm{mm} / \mathrm{s})$ & $23,37 \pm 0,9$ & $23,05 \pm 0,7$ & $22,0 \pm 0,72$ & $23,4 \pm 0,5 \#$ & $23,5 \pm 0,9$ & $26 \pm 1,5 \#$ \\
\hline & EF (\%) & $83 \pm 2$ & $82 \pm 62$ & $82 \pm 1,9$ & $87 \pm 1,5 \#$ & $84 \pm 1,5$ & $88 \pm 1,3 \#$ \\
\hline \multirow{10}{*}{$\begin{array}{c}\text { Diastolic } \\
\text { parameters }\end{array}$} & $E(\mathrm{~mm} / \mathrm{s})$ & $0,91 \pm 0,04$ & $0,94 \pm 0,04$ & $0,91 \pm 0,06$ & $1,05 \pm 0,07 \#$ & $0,831 \pm 0,03$ & $0,924 \pm 0,06$ \\
\hline & $A(\mathrm{~mm} / \mathrm{s})$ & $0,66 \pm 0,04$ & $0,78 \pm 0,05 \#$ & $0,79 \pm 0,08$ & $0,87 \pm 0,07$ & $0,59 \pm 0,05$ & $0,79 \pm 0,05 \#$ \\
\hline & E/A & $1,39 \pm 0,06$ & $1,22 \pm 0,08$ & $1,18 \pm 0,05$ & $1,22 \pm 0,04$ & $1,48 \pm 0,11$ & $1,2 \pm 0,06 \#$ \\
\hline & DT (ms) & $46,62 \pm 4,1$ & $34,12 \pm 2,5 \#$ & $48,5 \pm 3,8$ & $40,2 \pm 3,0$ & $53,4 \pm 3,8$ & $44,1 \pm 3,2 \#$ \\
\hline & DT/E & $51,91 \pm 4,9$ & $36,66 \pm 3,3 \#$ & $56,7 \pm 6,5$ & $40,39 \pm 4,8 \#$ & $65,21 \pm 5,3$ & $50,18 \pm 5,4 \#$ \\
\hline & E/DT & $21,28 \pm 3,2$ & $28,98 \pm 2,8 \#$ & $20,61 \pm 3,0$ & $27,51 \pm 2,6 \#$ & $16,53 \pm 1,7$ & $22,66 \pm 3,0$ \\
\hline & $\mathrm{e}^{\prime}(\mathrm{mm} / \mathrm{s})$ & $21,23 \pm 0,99$ & $24,73 \pm 0,61 \#$ & $22,94 \pm 1,0$ & $27,01 \pm 1,1 \#$ & $20,9 \pm 0,5$ & $23,8 \pm 0,9 \#$ \\
\hline & $\mathrm{a}^{\prime}(\mathrm{mm} / \mathrm{s})$ & $27,61 \pm 1,9$ & $31,35 \pm 1,5 \#$ & $28,72 \pm 2,2$ & $31,06 \pm 1,5$ & $25,9 \pm 1,9$ & $29,8 \pm 1,9$ \\
\hline & $e^{\prime} / a^{\prime}$ & $0,78 \pm 0,04$ & $0,79 \pm 0,03$ & $0,83 \pm 0,06$ & $0,88 \pm 0,04$ & $0,85 \pm 0,06$ & $0,82 \pm 0,04$ \\
\hline & $E / e^{\prime}$ & $43,6 \pm 2,6$ & $38,29 \pm 1,6 \#$ & $39,5 \pm 1,7$ & $38,61 \pm 1,5$ & $39,64 \pm 0,9$ & $38,95 \pm 2,3$ \\
\hline \multirow{4}{*}{$\begin{array}{l}\text { Overall cardiac } \\
\text { function }\end{array}$} & HR (bpm) & $266,2 \pm 9,8$ & $316,2 \pm 7,5 \#$ & $270 \pm 17$ & $271 \pm 10$ & $236 \pm 12$ & $257 \pm 11$ \\
\hline & SV (ml) & $0,71 \pm 0,04$ & $0,67 \pm 0,05$ & $0,702 \pm 0,05$ & $0,847 \pm 0,06 \#$ & $0,589 \pm 0,03$ & $0,601 \pm 0,04$ \\
\hline & $\mathrm{CO}(\mathrm{ml} / \mathrm{min})$ & $188,8 \pm 10,5$ & $211,2 \pm 13,2 \#$ & $187,7 \pm 13,7$ & $231,7 \pm 20,2 \#$ & $138,5 \pm 11,2$ & $155,6 \pm 14,4$ \\
\hline & $N$ & 8 & 8 & 10 & 10 & 10 & 10 \\
\hline
\end{tabular}




\section{Discussion}

In the present study, we have investigated effects of the istaroxime metabolite PST3093 at molecular, cellular and in-vivo levels. The interest in this molecule is motivated by 1) the possibility that it may actually contribute to (i.e. be endowed with) the unique mechanism of action and interesting therapeutic profile of istaroxime (inotropy and lusitropy at low proarrhythmic risk, confirmed in phase 2 clinical trials) $[15,16,19,20]$ and 2) the possibility it would afford to test the clinical benefit associated specifically with the rescue of SERCA2a depression, which is widely recognized as the basis for many among HF abnormalities.

PST3093 effect has been tested in three experimental settings with incremental level of biological organization, including in-vivo measurements from diseased hearts. The consistency of effects across these three sets of experiments confers robustness to the findings.

The results of molecular studies indicate that PST3093 differs from istaroxime because it is devoid of any inhibitory activity on the $\mathrm{Na}^{+} / \mathrm{K}^{+}$ATPase, while retaining SERCA2a stimulatory action. This identifies PST3093 as a "selective" SERCA2a activator. The results also indicate that, similar to istaroxime [28], PST3093 may act by weakening SERCA-PLN interaction. In rat preparations, PST3093 (and istaroxime) increased SERCA2a $\mathrm{V}_{\max }$. Albeit this apparently conflicts with the notion that interference with PLN should decrease the $\mathrm{K}_{d} \mathrm{Ca}$ instead [29], the same pattern coexisted with evidence, by several independent approaches, of istaroxime antagonism of SERCA2a-PLN interaction [28]. The reason for this apparent discrepancy is unclear; the observation that PST3093 effect on $\mathrm{SR} \mathrm{Ca}^{2+}$ uptake was present in rat cardiac myocytes (i.e. at physiological $\mathrm{Ca}^{2+}$ concentrations) suggests that it may reside in specificities of the microsomal preparation.

Investigations in intact ventricular myocytes confirm a negligible effect of PST3093 on $\mathrm{Na}^{+} / \mathrm{K}^{+}$ pump function. Furthermore, PST3093 abolished STZ-induced intracellular $\mathrm{Ca}^{2+}$ abnormalities likely dependent on SERCA2a downregulation. While PST3093 clearly affected several $\mathrm{Ca}^{2+}$ cycling parameters under $\mathrm{V}$-clamp conditions (Figure 6), it only reduced resting cytosolic $\mathrm{Ca}^{2+}$ 
level $\left(\mathrm{Ca}_{\text {rest }}\right)$ in field-stimulated myocytes (Figure 5). As we have previously shown in this experimental model [21], evaluation of $\mathrm{Ca}^{2+}$ handling without controlling membrane potential (field stimulation) and without disabling competing mechanisms (NCX) may be unsuitable to detect SERCA2a activation. This is why we also performed experiments under V-clamp and with disabled NCX, shown in Figure 6. Field-stimulation experiments are nonetheless informative because they better represent drug effects at the cell level under "physiological" conditions. PST3093 effect in field-stimulated myocytes are apparently small, the statistically significant one being limited to a reduction in $\mathrm{Ca}_{\text {rest }}$. However, this result indicates that PST3093 allows the other $\mathrm{Ca}^{2+}$ cycling parameters to be preserved (or even showing a trend to improve) at a lower cytosolic $\mathrm{Ca}^{2+}$ level. Considering that all $\mathrm{Ca}^{2+}$ homeostatic mechanisms are in place in this setting, this is precisely what should be expected from pure SERCA2a activation $[2,18]$, i.e. improved subcellular $\mathrm{Ca}^{2+}$ compartmentalization. That this apparently small change in myocyte physiology has an impact on in-vivo cardiac performance is shown by the in-vivo echo measurements (Figure 9).

The present in-vivo studies were conducted by echocardiography in a disease model characterized by impairment of SERCA2a function [21,30]. In this model, PST3093 infusion improved overall cardiac performance (SV and $\mathrm{CO}$ ); both systolic and diastolic indexes were positively affected by the agent. Mechanistic interpretation of echocardiographic indexes is often ambiguous. For instance, both DT prolongation and shortening have been associated with deterioration of diastolic function [19,31]. These puzzling observations can be interpreted by considering the contribution to DT of opposing factors, each prevailing in a specific condition [32]. At any rate, whenever HF was associated with DT shortening, istaroxime (having PST3093 as a metabolite) prolonged it [15,19]. A further difficulty may arise from the expectation that ino-lusitropy may increase atrial contraction (A amplitude) and ventricular relaxation (E amplitude) at the same time, thus conceivably making their ratio (E/A) unable to detect drug effects on diastolic function. An approach to the interpretation of drug effects, 
independent of mechanistic models, is to check whether the drug counters disease-induced abnormalities. In the case of PST3093, this was true for the majority of indexes (7 out of 11), the most notable exception being a small further increase in LVEDD. While increments in LVEDD are usually associated with deterioration of systolic function, PST3093 tended to decrease LVESD instead. The LVEDD increment was indeed associated with increased SV, and EF to which it likely contributed.

With the exception of guinea-pig, PST3093 efficacy on SERCA2a function in the diseased condition consistently contrasted with the lack of effect in healthy preparations (Figures 3 and 5). This suggests that SERCA2a function, while not strictly limiting in health, may become so whenever its "reserve" is diminished. This view may not clash with the clear-cut effect of PLN knock-out in healthy murine myocytes; indeed, SERCA2a modulation by PST3093 may be, albeit functionally significant, subtler than complete PLN ablation.

While failing to increase the amplitude of CaT, PST3093 improved echo indexes of systolic function. Mechanisms at two levels may account for this observation: at the intracellular level, increased compartmentation of $\mathrm{Ca}^{2+}$ within the SR may improve the energetic efficiency of $\mathrm{Ca}^{2+}$ cycling [33]; at the organ level, improved relaxation may increase preload, with its wellknown impact on systolic force [34]. Indeed, normalization of diastolic function in HF patients with preserved ejection fraction, may restore cardiac output irrespective of changes in the latter [35]. On the other hand, digoxin, whose mechanism of action is purely inotropic, accelerated early relaxation (DT shortening and e' increase). This is consistent with systo-diastolic coupling, i.e. the contribution to early relaxation of elastic restitution (recoil) of systolic force [36].

Beside affording inotropy and lusitropy, SERCA2a stimulation may improve intracellular $\mathrm{Ca}^{2+}$ compartmentalization, with potential long-term effects on energetic efficiency and biology of cardiac myocytes [2]. 
PST3093 is remarkably less toxic than istaroxime which, in turn, has a lower proarrhythmic risk as compared to digoxin [17]. We speculate that the low PST3093 toxicity, relative to istaroxime, may be due its failure to inhibit the $\mathrm{Na}^{+} / \mathrm{K}^{+}$pump. Absence of interaction with 50 cardiac and non-cardiac targets commonly involved in drug toxicity provides at least a first level evidence of PST3093 suitability as a therapeutic agent.

\subsection{Limitations}

Whereas, in the in-vivo experiments PST3093 effects generally achieved a maximum at $30 \mathrm{~min}$ of infusion, istaroxime infusion period was limited to $15 \mathrm{~min}$. Our previous study [21] indicates that a 15 min infusion is sufficient for modulation of diastolic parameters by istaroxime. This time-point was selected in the present study to minimize metabolism to PST3093, thus allowing it to differentiate istaroxime's own effect from that of its metabolite. Nonetheless, istaroxime effects reported here might differ from the steady-state ones, described in previous studies $[15,16,19]$, to which PST3093 (the metabolite) might actually contribute.

Translation of the present in-vivo results to human therapy has to consider differences between clinical HF and the STZ rat model, which has specific hemodynamic features [37]. However, consistency of the istaroxime effect reported here with that described in HF patients [16] supports this translation.

\subsection{Therapeutic relevance and perspective}

The results of this study identify PST3093 as a prototype "selective" (i.e. devoid of $\mathrm{Na}^{+} / \mathrm{K}^{+}$ pump inhibition) SERCA2a activator. This may entail significant differences from the already characterized pharmacodynamic profile of istaroxime.

In the case of istaroxime, lack of the proarrhythmic effect (expected from $\mathrm{Na}^{+} / \mathrm{K}^{+}$ATPase inhibition [14]) is likely due to SERCA2a stimulation. Indeed, the latter may reduce the occurrence of " $\mathrm{Ca}^{2+}$ waves" and the resulting "triggered activity"[2,38,39]. It is logical to 
predict that a pure SERCA2a activator may exert substantial antiarrhythmic effects, at least under the common conditions characterized by SR instability (e.g. HF). On the other hand, $\mathrm{Na}^{+} / \mathrm{K}^{+}$pump inhibition may contribute to inotropy; thus, at least theoretically, PST3093 should increase systolic force less than istaroxime. The present results argue for a PST3093 effect on global cardiac function, including positive inotropy. Moreover, compared to istaroxime, PST3093 has a much longer half-life that, per se, may also prolong the beneficial hemodynamic effect of istaroxime infusion.

\subsection{Conclusions}

HF treatment would strongly benefit from the availability of ino-lusitropic agents with a favorable profile. PST3093 is the main metabolite of istaroxime showing a longer half-life in human circulation compared to parent drug, activates SERCA2a, doesn't inhibit $\mathrm{Na}^{+}-\mathrm{K}^{+}$ ATPase and improves systolic and diastolic performance in a model of diabetic cardiomyopathy. Overall, PST3093 acting as "selective" SERCA2a activator can be considered the prototype of a novel pharmacodynamic class for the ino-lusitropic approach of HF. After more than 50 years from the suggestion of the involvement of a reduced SERCA2a function as a cause of the depressed cardiac function and the increased arrhythmias in HF, we may have the possibility to prove this hypothesis and provide a "causal" and selective therapy for HF patients.

\section{Declaration of interest}

$\mathrm{MF}$ and $\mathrm{PB}$ are Windtree employees, $\mathrm{PF}$ and $\mathrm{GB}$ are Windtree consultants, $\mathrm{S}-\mathrm{CH}$ is an employee of CVie Therapeutics Limited. All the other Authors declare no conflict of interest.

\section{Funding}


This work was supported by CVie Therapeutics Limited (Taipei, Taiwan), WindTree Therapeutics (Warrington, USA) and University of Milano Bicocca.

\section{Supplementary material}

Supplementary data associated with this article are reported in the Online Supplement.

\section{References}

[1] D.M. Bers, S. Despa, Cardiac myocytes $\mathrm{Ca} 2+$ and $\mathrm{Na}+$ regulation in normal and failing hearts, J. Pharmacol. Sci. 100 (2006) 315-322. https://doi.org/10.1254/jphs.CPJ06001X.

[2] A. Zaza, M. Rocchetti, Calcium Store Stability as an Antiarrhythmic Endpoint, Curr. Pharm. Des. 21 (2015) 1053-1061. https://doi.org/10.2174/1381612820666141029100650.

[3] H. Nakayama, X. Chen, C.P. Baines, R. Klevitsky, X. Zhang, H. Zhang, N. Jaleel, B.H.L. Chua, T.E. Hewett, J. Robbins, S.R. Houser, J.D. Molkentin, Ca2+- and mitochondrial-dependent cardiomyocyte necrosis as a primary mediator of heart failure, J. Clin. Invest. 117 (2007) 2431-2444. https://doi.org/10.1172/JCI31060.

[4] J. SUKO, J.H.K. VOGEL, C.A. CHIDSEY, Intracellular Calcium and Myocardial Contractility, Circ. Res. 27 (1970) 235-247. https://doi.org/10.1161/01.res.27.2.235.

[5] P. V. Sulakhe, N.S. Dhalia, Excitation-contraction coupling in heart. VII. Calcium accumulation in subcellular particles in congestive heart failure., J. Clin. Invest. 50 (1971) 1019-1027. https://doi.org/10.1172/JCI106573.

[6] E.G. Kranias, R.J. Hajjar, Modulation of cardiac contractility by the phopholamban/SERCA2a regulatome, Circ. Res. 110 (2012) 1646-1660. 
https://doi.org/10.1161/CIRCRESAHA.111.259754.

[7] J.K. Gwathmey, L. Copelas, R. MacKinnon, F.J. Schoen, M.D. Feldman, W.

Grossman, J.P. Morgan, Abnormal intracellular calcium handling in myocardium from patients with end-stage heart failure, Circ. Res. 61 (1987) 70-76.

https://doi.org/10.1161/01.RES.61.1.70.

[8] M. Arai, N.R. Alpert, D.H. MacLennan, P. Barton, M. Periasamy, Alterations in sarcoplasmic reticulum gene expression in human heart failure: A possible mechanism for alterations in systolic and diastolic properties of the failing myocardium, Circ. Res. 72 (1993) 463-469. https://doi.org/10.1161/01.RES.72.2.463.

[9] K. Haghighi, A.G. Schmidt, B.D. Hoit, A.G. Brittsan, A. Yatani, J.W. Lester, J. Zhai, Y. Kimura, G.W. Dorn, D.H. MacLennan, E.G. Kranias, Superinhibition of Sarcoplasmic Reticulum Function by Phospholamban Induces Cardiac Contractile Failure, J. Biol. Chem. 276 (2001) 24145-24152. https://doi.org/10.1074/jbc.M102403200.

[10] F. Del Monte, S.E. Harding, G.W. Dec, J.K. Gwathmey, R.J. Hajjar, Targeting phospholamban by gene transfer in human heart failure, Circulation. 105 (2002) 904907. https://doi.org/10.1161/hc0802.105564.

[11] C. Kho, A. Lee, D. Jeong, J.G. Oh, A.H. Chaanine, E. Kizana, W.J. Park, R.J. Hajjar, SUMO1-dependent modulation of SERCA2a in heart failure, Nature. 477 (2011) 601606. https://doi.org/10.1038/nature10407.

[12] M. Kaneko, H. Yamamoto, H. Sakai, Y. Kamada, T. Tanaka, S. Fujiwara, S. Yamamoto, H. Takahagi, H. Igawa, S. Kasai, M. Noda, M. Inui, T. Nishimoto, A pyridone derivative activates SERCA2a by attenuating the inhibitory effect of phospholamban, Eur. J. Pharmacol. 814 (2017) 1-8. 
[13] T.M. Schaaf, E. Kleinboehl, S.L. Yuen, L.N. Roelike, B. Svensson, A.R. Thompson, R.L. Cornea, D.D. Thomas, Live-Cell Cardiac-Specific High-Throughput Screening Platform for Drug-Like Molecules That Enhance Ca2+ Transport, 9 (2020).

[14] M. Rocchetti, A. Besana, G. Mostacciuolo, R. Micheletti, P. Ferrari, S. Sarkozi, C. Szegedi, I. Jona, A. Zaza, Modulation of sarcoplasmic reticulum function by $\mathrm{Na}+\mathrm{K}+$ pump inhibitors with different toxicity: Digoxin and PST2744 [(E,Z)-3-((2aminoethoxy)imino)androstane-6,17-dione hydrochloride], J. Pharmacol. Exp. Ther. 313 (2005) 207-215. https://doi.org/10.1124/jpet.104.077933.

[15] S.J. Shah, J.E.A. Blair, G.S. Filippatos, C. MacArie, W. Ruzyllo, J. Korewicki, S.I. Bubenek-Turconi, M. Ceracchi, M. Bianchetti, P. Carminati, D. Kremastinos, J. Grzybowski, G. Valentini, H.N. Sabbah, M. Gheorghiade, Effects of istaroxime on diastolic stiffness in acute heart failure syndromes: Results from the Hemodynamic, Echocardiographic, and Neurohormonal Effects of Istaroxime, a Novel Intravenous Inotropic and Lusitropic Agent: A Randomized Controlled Trial in P, Am. Heart J. 157 (2009) 1035-1041. https://doi.org/10.1016/j.ahj.2009.03.007.

[16] V. Carubelli, Y. Zhang, M. Metra, C. Lombardi, G.M. Felker, G. Filippatos, C.M. O’Connor, J.R. Teerlink, P. Simmons, R. Segal, G. Malfatto, M.T. La Rovere, D. Li, X. Han, Z. Yuan, Y. Yao, B. Li, L.F. Lau, G. Bianchi, J. Zhang, Treatment with 24 hour istaroxime infusion in patients hospitalised for acute heart failure: a randomised, placebo-controlled trial, Eur. J. Heart Fail. 22 (2020) 1684-1693. https://doi.org/10.1002/ejhf.1743.

[17] R. Micheletti, G.G. Mattera, M. Rocchetti, A. Schiavone, M.F. Loi, A. Zaza, R.J.P. Gagnol, S. De Munari, P. Melloni, P. Carminati, G. Bianchi, P. Ferrari, Pharmacological profile of the novel inotropic agent (E,Z)-3-((2aminoethoxy)imino)androstane-6,17-dione hydrochloride (PST2744), J. Pharmacol. 
Exp. Ther. 303 (2002) 592-600. https://doi.org/10.1124/jpet.102.038331.

[18] M. Alemanni, M. Rocchetti, D. Re, A. Zaza, Role and mechanism of subcellular Ca2+ distribution in the action of two inotropic agents with different toxicity, J. Mol. Cell. Cardiol. 50 (2011) 910-918. https://doi.org/10.1016/j.yjmcc.2011.02.008.

[19] H.N. Sabbah, M. Imai, D. Cowart, A. Amato, P. Carminati, M. Gheorghiade, Hemodynamic Properties of a New-Generation Positive Luso-Inotropic Agent for the Acute Treatment of Advanced Heart Failure, Am. J. Cardiol. 99 (2007) 41A-46A. https://doi.org/10.1016/j.amjcard.2006.09.005.

[20] M. Gheorghiade, J.E.A. Blair, G.S. Filippatos, C. Macarie, W. Ruzyllo, J. Korewicki, S.I. Bubenek-Turconi, M. Ceracchi, M. Bianchetti, P. Carminati, D. Kremastinos, G. Valentini, H.N. Sabbah, Hemodynamic, Echocardiographic, and Neurohormonal Effects of Istaroxime, a Novel Intravenous Inotropic and Lusitropic Agent. A Randomized Controlled Trial in Patients Hospitalized With Heart Failure, J. Am. Coll. Cardiol. 51 (2008) 2276-2285. https://doi.org/10.1016/j.jacc.2008.03.015.

[21] E. Torre, M. Arici, A.M. Lodrini, M. Ferrandi, P. Barassi, S.-C. Hsu, G.-J. Chang, E. Boz, E. Sala, S. Vagni, C. Altomare, G. Mostacciuolo, C. Bussadori, P. Ferrari, G. Bianchi, M. Rocchetti, SERCA2a stimulation by istaroxime improves intracellular $\mathrm{Ca} 2+$ handling and diastolic dysfunction in a model of diabetic cardiomyopathy, Cardiovasc. Res. (2021). https://doi.org/10.1093/cvr/cvab123.

[22] M. Valero-Muñoz, W. Backman, F. Sam, Murine Models of Heart Failure With Preserved Ejection Fraction: A “Fishing Expedition,” JACC Basic to Transl. Sci. 2 (2017) 770-779. https://doi.org/10.1016/j.jacbts.2017.07.013.

[23] R. Micheletti, F. Palazzo, P. Barassi, G. Giacalone, M. Ferrandi, A. Schiavone, B. Moro, O. Parodi, P. Ferrari, G. Bianchi, Istaroxime, a Stimulator of Sarcoplasmic Reticulum Calcium Adenosine Triphosphatase Isoform 2a Activity, as a Novel 
Therapeutic Approach to Heart Failure, Am. J. Cardiol. 99 (2007) 24A-32A. https://doi.org/10.1016/j.amjcard.2006.09.003.

[24] M. Rocchetti, A. Besana, G. Mostacciuolo, P. Ferrari, R. Micheletti, A. Zaza, Diverse toxicity associated with cardiac $\mathrm{Na}+\mathrm{K}+$ pump inhibition: Evaluation of electrophysiological mechanisms, J. Pharmacol. Exp. Ther. 305 (2003) 765-771. https://doi.org/10.1124/jpet.102.047696.

[25] K.E. Odening, A.-M. Gomez, D. Dobrev, L. Fabritz, F.R. Heinzel, M.E. Mangoni, C.E. Molina, L. Sacconi, G. Smith, M. Stengl, D. Thomas, A. Zaza, C.A. Remme, J. Heijman, ESC working group on cardiac cellular electrophysiology position paper: relevance, opportunities, and limitations of experimental models for cardiac electrophysiology research, EP Eur. 23 (2021) 1795-1814. https://doi.org/10.1093/europace/euab142.

[26] C. Altomare, C. Bartolucci, L. Sala, J. Bernardi, G. Mostacciuolo, M. Rocchetti, S. Severi, A. Zaza, IKr Impact on Repolarization and Its Variability Assessed by Dynamic Clamp, Circ. Arrhythmia Electrophysiol. 8 (2015) 1265-1275. https://doi.org/10.1161/CIRCEP.114.002572.

[27] R.M. Lang, M. Bierig, R.B. Devereux, F.A. Flachskampf, E. Foster, P.A. Pellikka, M.H. Picard, M.J. Roman, J. Seward, J. Shanewise, S. Solomon, K.T. Spencer, M. St John Sutton, W. Stewart, American Society of Echocardiography's Nomenclature and Standards Committee; Task Force on Chamber Quantification; American College of Cardiology Echocardiography Committee; American Heart Association; European Association of Echocardiography; European So, Eur J Echocardiogr. 7 (2006) 79-108.

[28] M. Ferrandi, P. Barassi, F. Tadini-Buoninsegni, G. Bartolommei, I. Molinari, M.G. Tripodi, C. Reina, M.R. Moncelli, G. Bianchi, P. Ferrari, Istaroxime stimulates SERCA2a and accelerates calcium cycling in heart failure by relieving phospholamban 
inhibition, Br. J. Pharmacol. 169 (2013) 1849-1861.

https://doi.org/10.1111/bph.12278.

[29] A.G. Brittsan, K.S. Ginsburg, G. Chu, A. Yatani, B.M. Wolska, A.G. Schmidt, M. Asahi, D.H. MacLennan, D.M. Bers, E.G. Kranias, Chronic SR Ca2+-ATPase inhibition causes adaptive changes in cellular Ca2+ transport, Circ. Res. 92 (2003) 769-776. https://doi.org/10.1161/01.RES.0000066661.49920.59.

[30] K.M. Choi, Y. Zhong, B.D. Hoit, I.L. Grupp, H. Hahn, K.W. Dilly, S. Guatimosim, W. Jonathan Lederer, M.A. Matlib, Defective intracellular Ca2+ signaling contributes to cardiomyopathy in type 1 diabetic rats, Am. J. Physiol. - Hear. Circ. Physiol. 283 (2002) H1398-H1408. https://doi.org/10.1152/ajpheart.00313.2002.

[31] M. Eren, S. Gorgulu, N. Uslu, S. Celik, B. Dagdeviren, T. Tezel, Relation between aortic stiffness and left ventricular diastolic function in patients with hypertension, diabetes, or both, Heart. 90 (2004) 37-43. https://doi.org/10.1136/heart.90.1.37.

[32] S. Mossahebi, S. Zhu, S.J. Kovács, Fractionating E-wave deceleration time into its stiffness and relaxation components distinguishes pseudonormal from normal filling, Circ. Cardiovasc. Imaging. 8 (2015). https://doi.org/10.1161/CIRCIMAGING.114.002177.

[33] T.R. Shannon, G. Chu, E.G. Kranias, D.M. Bers, Phospholamban Decreases the Energetic Efficiency of the Sarcoplasmic Reticulum Ca Pump, J. Biol. Chem. 276 (2001) 7195-7201. https://doi.org/10.1074/jbc.M007085200.

[34] H.A. Shiels, E. White, The Frank-Starling mechanism in vertebrate cardiac myocytes, J. Exp. Biol. 211 (2008) 2005-2013. https://doi.org/10.1242/jeb.003145.

[35] T. Tobushi, M. Nakano, K. Hosokawa, H. Koga, A. Yamada, Improved diastolic function is associated with higher cardiac output in patients with heart failure irrespective of left ventricular ejection fraction, J. Am. Heart Assoc. 6 (2017). 
https://doi.org/10.1161/JAHA.116.003389.

[36] A.T. Burns, A. La Gerche, D.L. Prior, A.I. MacIsaac, Left Ventricular Untwisting Is an Important Determinant of Early Diastolic Function, JACC Cardiovasc. Imaging. 2 (2009) 709-716. https://doi.org/10.1016/j.jcmg.2009.01.015.

[37] M.J. Mihm, J.L. Seifert, C.M. Coyle, J.A. Bauer, Diabetes related cardiomyopathy time dependent echocardiographic evaluation in an experimental rat model, Life Sci. 69 (2001) 527-542. https://doi.org/10.1016/S0024-3205(01)01141-9.

[38] Y. Bai, P.P. Jones, J. Guo, X. Zhong, R.B. Clark, Q. Zhou, R. Wang, A. Vallmitjana, R. Benitez, L. Hove-Madsen, L. Semeniuk, A. Guo, L.S. Song, H.J. Duff, S.R.W. Chen, Phospholamban knockout breaks arrhythmogenic Ca2+ waves and suppresses catecholaminergic polymorphic ventricular tachycardia in mice, Circ. Res. 113 (2013) 517-526. https://doi.org/10.1161/CIRCRESAHA.113.301678.

[39] M. Fernandez-Tenorio, E. Niggli, Stabilization of Ca2+ signaling in cardiac muscle by stimulation of SERCA, J. Mol. Cell. Cardiol. 119 (2018) 87-95. https://doi.org/10.1016/j.yjmcc.2018.04.015. 